\title{
Modèle didactique proposé basé sur la pédagogie différenciée pour développer les compétences de l'enseignement de la compréhension orale chez les futurs enseignants du FLE
}

\section{Dr. Nesrine Salah Abdel Ghany}

L'enseignement de l'oral se heurte à des obstacles didactiques: c'est un domaine dans lequel les professeurs ne disposent pas de références communes. Pour enseigner les pratiques orales efficaces, les professeurs manquent de «modèles» auxquels ils puissent aisément se reporter, et malgré sa place centrale, l'oral ne fait que rarement l'objet d'une programmation fondée sur une progression et la complexification progressive des tâches, aussiun large foyer entre ce qui s'enseigne dans nos facultés de pédagogie et ce qui se déroule dans les classes de langues dans les écoles gouvernementales, les méthodes classiques adoptées dans l'apprentissage des compétences de la langue, et l'insuffisance des ateliers de travail, se reflètent négativement sur le niveau de l'acquisition de la langue française des étudiants au cycle secondaire, Nos étudiantes n'arrivent ni à bien parler ni à bien enseigner, elles ont des difficultés surtout dans l'apprentissage de la compréhension orale,c'est ce qu'on vit avec les étudiantes pendant plus de 10 ans de l'enseignement de la méthodologie française au Faculté de Jeunes Filles. Pour contribuer à l'élaboration d'un modèle didactique dans ce domaine, on proposera de réfléchir à un modèle opérationnel, suffisamment simple, cohérent et rentable pour engendrer une pratique pédagogique concernant l'apprentissage et l'enseignement de l'oral.Ce qui a mener la chercheuse à présenter aux étudiantesun guide; un patron,un modèle à suivre pour développer les compétences de l'enseignement de la compréhension orale basé sur la pédagogie différenciée pour les aider à pouvoir pratiquer cette approche qui désigne "l'effort de diversification".

I- Introduction : La langue est un moyen de communication : c'est un instrument à travers lequel l'homme peut exprimer ses idées, ses gouts, ses sentiments, ses besoins et ses opinions. Elle facilite l'interaction entre les individus de la même société et entre ceux des différentes sociétés. Par conséquent, elle reflète la culture et la civilisation de chaque pays. C'est pourquoi apprendre les langues étrangères nécessite de reconnaître la culture, les coutumes et les habitudes des autres communautés afin de parvenir à communiquer.

La communication est la finalité essentielle de l'apprentissage d'une langue étrangère, or tout acte de communication suppose l'existence d'un émetteur et d'un récepteur même virtuel, la compréhension d'un

\section{3}


message oral est donc indispensable, car il faut comprendre le message pour pouvoir y réagir et y répondre. Longtemps cette activité de compréhension a été négligée parce qu'on avait l'impression que l'étudiant en position d'écoute était passif. Comprendre, implique des opérations mentales complexes qui ne sont pas immédiatement perceptibles ; mais l'étudiant est aussi actif en réception qu'en production.

L'enseignement de l'oral a toujours fait l'objet de préoccupations pédagogiques et linguistiques chez les enseignants de français langue maternelle et secondaire. En effet, ceux-ci éprouvent un grand inconfort devant l'oral et son enseignement.

Il est tout à fait justifié d'affirmer que l'oral est l'élément le moins considéré de l'enseignement du français. Les principaux facteurs responsables de cette situation problématique sont de divers ordres : difficulté des enseignants à objectiver et à évaluer les performances des étudiants, inconfort manifeste devant l'enseignement et l'évaluation de l'oral, absence de matériel didactique adapté, utilisation de méthodes d'enseignement intuitives, manque de précision dans les objectifs généraux des programmes d'études, l'absence des ateliers d'apprentissage à caractère formatif ", visant à consolider les acquis des étudiants et aussi à les préparer adéquatement à affronter un examen sommatif de communication orale.

Wirthner $^{1}$ (1991) affirme que l'enseignement de l'oral est encore lacunaire, car il manque d'indications et d'objectifs méthodologiques précis. Lazure (1994) souligne cette lacune à laquelle les enseignants de français sont confrontés en précisant qu'il y a très peu d'indications sur les critères de progression des apprentissages oraux dans les programmes d'études.

L'oral est une des bêtes noires de l'enseignement du français. Afin de remédier les problèmes précédemment évoqués et pour donner à l'oral sa place comme objet d'enseignement, Schneuwly (1996) précise que l'enseignement de l'oral doit porter d'abord et avant tout sur des formes bien définies et régulées de l'extérieur, telles que des situations de

${ }^{1}$ Wirthner, M. (1991). L'oral comme expérience de soi et rapport à l'autre. In M. Wirthner, D. Martin et P. Perrenoud. Paris. 
communication publique. Il faut faire entendre aux étudiants la langue orale telle que parlée au quotidien et il faut se pencher sur différentes variantes et contextes de diffusion des situations d'oral : radio, télévision, cassettes, disques, etc.

La compréhension orale est une des étapes les plus fondamentales de la communication et dans l'acquisition d'une langue étrangère. L'écoute dans des situations authentiques permet à l'étudiant de se familiariser avec les sons de la langue non maternelle, elle permet de présenter à l'apprenant des discours plutôt que des phrases, elle amène l'étudiant à s'appuyer sur les éléments du texte et les connaissances personnelles pour comprendre un message oral.

L'écoute n'est pas un savoir sur lequel les manuels scolaires s'étendent beaucoup. Cette compétence est souvent délaissée par les enseignants qui pensent que les étudiants l'acquièrent automatiquement quand ils apprennent à parler une langue étrangère. De plus ils croient enseigner la compréhension de l'oral alors qu'en réalité ce qui est fait n'est autre que de l'expression orale ou de la compréhension de l'écrit. On parle beaucoup de la compréhension du français oral mais on n'en fait pas un objet d'apprentissage spécifique.

L'enseignement de l'oral se heurte à des obstacles didactiques : c'est un domaine dans lequel les professeurs ne disposent pas de références communes. Pour enseigner les pratiques orales efficaces, les professeurs manquent de «modèles» auxquels ils puissent aisément se reporter $»^{2}$, la pédagogie de l'oral est plus difficile à mettre en œuvre que celle de l'écrit dans le quotidien de la classe. Les exercices semblent se ressembler des cycles primaires au collège (réciter, exposer, questionner, interroger, répondre, débattre) alors qu'ils devraient être différenciés dans la complexité des tâches proposées selon les possibilités des étudiants. Or, malgré sa place centrale, l'oral ne fait que rarement l'objet d'une programmation fondée sur une progression et la complexification progressive des tâches.

Il ne fait pas assez souvent l'objet d'évaluation explicite. ${ }^{3} \mathrm{Ce}$ qui se coïncide avec l'expérience de la chercheuse, car pendant plus de 15 ans

${ }^{2}$ Rapport de l'Inspection Générale, La place de l'oral dans les enseignements : de l'école primaire au lycée, 1999, p. 3.

3 L'ORAL : langage, situations, références. Rapport de 1'IGEN n 99-023, La place de l'oral dans les enseignements : de l'école primaire au lycée, Septembre 1999.

\section{5}


de l'observation des stagiaires durant leurs stages pratiques à la 3ème et à la 4ème année scolaire du département de la langue française pédagogique Faculté des Jeunes filles, on peut avouer qu'il y a un large foyer entre ce qui s'enseigne dans nos facultés de pédagogie et ce qui se déroule dans les classes de langues dans les écoles gouvernementales.

On peut signaler que les méthodes classiques adoptées dans l'apprentissage des compétences de la langue, et l'insuffisance des ateliers de travail (qui assurent l'entrainement des compétences de l'enseignement), se reflètent négativement sur le niveau de l'acquisition de la langue française des étudiants au cycle secondaire.

Nos étudiantes n'arrivent ni à bien parler ni à bien enseigner, elles ont des difficultés surtout dans l'apprentissage de la compréhension orale g c'est ce que nous vivons avec nos étudiantes pendant plus de 10 ans de l'enseignement de la méthodologie française au Faculté de Jeunes Filles.

Pour contribuer à l'élaboration d'un modèle didactique dans ce domaine, on proposera de réfléchir à un modèle opérationnel, suffisamment simple, cohérent et rentable pour engendrer une pratique pédagogique concernant l'apprentissage et l'enseignement de l'oral.

Pourquoi un modèle Le postulat visé ici est de présenter à nos étudiantes un guide; un patron à suivre dans leur stage pratique afin de mener à bien leur pratique de l'enseignement de la langue française soit l'orale ou l'écrite.

Le bon Dieu nous a créé différents ; on se diffère dans l'ethnie, le sexe; la forme, les attitudes, le couleur; la religion, et la façon d'apprendre; l'être humain même se diffère d'un moment à un autre; Confucius le philosophe chinois considéré comme le premier "éducateur» de la

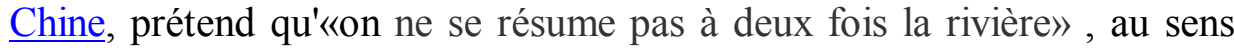
qu'on ne pratique pas le même acte deux fois parce que l'homme se diffère d'un temps à autre et l'eau du rivière aussi se change car elle n'est pas stable.

Les différences sont naturelles et nécessaires à l'évolution. Diversité et diversification sont des caractéristiques du vivant. Le changement c'est la loi de la vie, et le processus éducatif ne diffère pas de la vie, l'enseignant et l'apprenant ont besoin eux aussi de se changer,

\section{6}


Burns $^{4}$ signale qu'il n'y a pas 2 apprenants qui progressent à la même vitesse; qui soient prêts à apprendre en même temps; qui utilisent les mêmes techniques d'étude; qui résolvent les problèmes exactement de la même manière; qui possèdent le même répertoire de comportements; qui possèdent le même profil d'intérêt; et qui soient motivés pour atteindre les mêmes buts.

Cependant les lois et les programmes officiels, donnent pour mission à l'enseignant de faire acquérir à tous ses étudiants un ensemble défini de compétences disciplinaires, tout en respectant les différences de chacun. Il revient alors à l'enseignant de tenter de répondre au problème de l'hétérogénéité dans les apprentissages en mettant en œuvre une différenciation pédagogique.

Alors, peut-on demander si nos enseignants réagissent à ses postulats ; différent-ils leurs méthodes d'enseignements; varient-ils dans leurs contenus; modifient-ils leurs systèmes d'évaluation; est ce que notre système éducatif donne la manière à nos enseignants pour choisir les méthodes les plus adaptées aux caractéristiques individuelles et aux besoins spécifiques de leurs étudiants!!!

Est- ce que nos futurs enseignants sachent comment traiter les différences entre leurs étudiants? Comment ne pas transformer les différences individuelles en inégalités de réussite scolaire reproduisant des inégalités sociales ?

Est-ce qu'ils peuvent aider leurs étudiants à mieux apprendre en prenant appui sur leurs différences? Lequel parmi eux croit que la différence est une valeur en soi, et que, c'est par les différences qu'on s'enrichit?

La pratique des étudiants (par suite les futurs enseignants) se caractérise par une uniformité certaine, voire une standardisation; elle repose sur l'image d'un étudiant unique, d'un type d'étudiant et ils enseignent donc de la même manière à tout le monde.

4 (Molette, 2010) La différenciation pédagogique en maternelle article support de l'animation pédagogique jeudi 11 février 2010, par Valérie Molette.

5 BRAILLON Anne La différenciation pédagogique. Pour l'implication des étudiants Institut Supérieur de Formation Pédagogique. 2008-2009.

\section{7}


L'enseignant doit être convaincu qu'il est différent, alors il fonctionne différemment, et ses étudiants aussi ; lui et ses étudiants ont des styles d'apprentissage différents.

En tant que professeur, Hubert ${ }^{6}$ réclame " plus je varierai la manière dont je présente le savoir à acquérir, plus je varierai les supports d'apprentissage, les supports d'évaluation, plus j'aurai de chances que les étudiants se retrouvent, soient à l'aise un jour ou l'autre par rapport à leur propre profil d'apprentissage".

Nous ne pouvons en effet aller à l'encontre de ce constat: nos étudiants sont différents entre eux alors ils ont certainement besoin d'un enseignement adapté à leurs particularités, tout cela nous encourage à envisager le thème de la différenciation pédagogique ; thème tellement négligé dans nos écoles.

Maintenant que nous avons examiné le problème de la faiblesse de nos étudiantes dans l'enseignement des compétences orales surtout la compréhension orale, ainsi que d'avoir un manque de connaissances des théories de la différenciation pédagogique, demeure une question qui se pose maintenant : Y a-t-il un modèle à suivre pour développer les compétences de l'enseignement de la compréhension orale basé sur la pédagogie différenciée pour aider nos enseignants à pouvoir pratiquer cette approche qui désigne "l'effort de diversification" .

II-Problématique de la recherche :La problématique de la recherche réside dans l'inefficacité des méthodes classiques de l'apprentissage de la langue dans le développement des compétences didactique et surtout les compétences orales de la langue française et l'incapacité de nos futurs enseignants à pratiquer la théorie de la différenciation pédagogique; ce qui a mené la chercheuse à poser cette question :

Quel est le modèle proposé pour développer les compétences de l'enseignement de l'oral chez les futurs enseignants du FLE selon la différenciation pédagogique.

De cette question dérivent les questions suivantes :

1. Quelles sont les compétences didactiques nécessaires pour enseigner la compréhension orale selon la différenciation pédagogique?

${ }^{6}$ HUBERT, J.-C. (s.d.). DE LA PEDAGOGIE DIFFERENCIEE. Consulté le juin 12, 2011, sur WWW.Spirale-edu-revue.fr/.../HUBERT_Jean-Claude_De_la_pedagogie_...

\section{8}


2. Dans quelle mesure les futurs enseignants du FLE sont dotés de ces compétences didactiques?

3. Quel est le modèle didactique proposé pour développer les compétences de l'enseignement de la compréhension orale selon la différenciation pédagogique?

4. Quelle est l'efficacité de ce modèle sur le développement des compétences de l'enseignement de l'oral chez les futurs enseignants du FLE?

III- Buts de la recherche : Cette recherche vise à :

1. Déterminer les compétences didactiques de l'enseignement des compétences de la compréhension orale selon la différenciation pédagogique.

2. Elaborer un modèle pour développer les compétences didactiques de la compréhension de l'oral selon la différenciation pédagogique.

3. Développer les compétences didactiques et linguistiques concernant l'enseignement de la compréhension de l'oral des futurs enseignants de Faculté des Jeunes Filles selon la différenciation pédagogique.

4. Présenter un modèle didactique basé sur la différenciation pédagogique pour enseigner la compréhension orale.

5. Entraîner les futurs enseignants à formuler les objectifs opérationnels.

6. Entraîner les futurs enseignants à gérer la classe tout en travaillant par groupe.

7. Envisager plusieurs pistes d'exploitation pédagogiques selon les Intelligences Multiples.

IV- Limites de la recherche : Cette recherche se limite à:

1. La langue française comme $2^{\text {ème }}$ langue étrangère.

2. Le premier terme de l'année scolaire $2011 / 2012$.

3. Les futurs enseignants de la $4^{\text {ème }}$ année pédagogique de Faculté de Jeunes Filles à l'Université d 'Ain Shams.

4. Les compétences didactiques concernant l'enseignement de la compréhension orale.

5. L'approche de la différenciation pédagogique.

V-Outils de la recherche :

1. Questionnaire pour déterminer les préférences concernant les sujets traités dans l'enseignement des compétences orales.

2. Test afin de mesurer les Intelligences Multiples des futurs enseignants de Faculté de Jeunes Filles.

\section{9}


3. Liste des compétences de l'enseignement de la compréhension orale selon la différenciation pédagogique.

4. Modèle didactique basé sur la différenciation pédagogique pour enseigner la compréhension orale.

5. Pré-post test afin de mesurer :

- les compétences didactiques de l'enseignement de la compréhension orale avant la pratique du programme,

- l'efficacité du modèle proposé,

- le développement des compétences de la compréhension orale après l'application du modèle proposé.

6. Grille d'observation pour évaluer les performances des futurs enseignants dans les compétences suivantes:

- Formulation des objectifs.

- Formation des équipes de travaille.

- Choix des multimédia pour la préparation de la leçon.

7. Fiche d'exploitation afin de préparer les leçons de la compréhension orale.

VI- Terminologie de la recherche:

1. Modèle didactique : on vise par «modèle didactique » toutes les étapes à suivre par nos futurs enseignants pour développer leur enseignement de la compréhension orale.

2. Les compétences: Les compétences sont l'ensemble des connaissances, des habiletés et des dispositions qui permettent d'agir, ${ }^{7}$

3. Les compétences didactiques: c'est l'ensemble des habilitées et des performances que possèdent l'étudiant- maîtres pour enseigner la compréhension orale soit sur le niveau de planification, d'exécution, ou d'évaluation de ses cours dans une situation d'enseignement.

4. La compréhension orale: La compréhension orale est un processus d'interprétation interactif au cours duquel les auditeurs font appel à la fois à leurs acquis et à leurs connaissances linguistiques pour comprendre le message, c'est une compétence qui vise à faire acquérir progressivement à l'apprenant des stratégies d'écoute premièrement et de compréhension d'énoncés à l'oral deuxièmement. Il ne s'agit pas d'essayer de tout faire comprendre aux apprenants, qui ont tendance à demander une définition pour chaque mot. 8

${ }^{7}$ Conseil de l'Europe. (2000). Un Cadre Europeen Commun de Reference pour les Langues. Strasbourg: Didier.

8 DUCROT- Jean Michèle (2005) : L'enseignement de la compréhension orale, Didactique de l'oral, du discours en FLE.

\section{0}


5. La différenciation pédagogique: 9 C'est l' action de la chercheuse en étant un des pédagogues qui, sur la base d'une solide connaissance des préalables et caractéristiques de ses étudiantes (futurs enseignants), et de formules pédagogiques et d'interventions diversifiées, du programme de formation et de l'environnement d'apprentissage, tend à harmoniser les différentes composantes d'une situation pédagogique ainsi que les relations entre elles, dans le but de favoriser l'apprentissage et de développer les compétences de l'enseignement de l'oral chez les étudiantes-maitres de la 4ieme année de la section française au Faculté de Jeunes Filles Université de Ain Shams

\section{VII- Procédures de la recherche:}

1. Déterminer les compétences de l'enseignement de la compréhension orale nécessaires aux futurs enseignants selon la différenciation pédagogique selon :

- Les études antérieures concernant l'enseignement de l'oral, la différenciation pédagogique et les modèles didactiques.

- L'élaboration d'une liste des compétences de l'enseignement de la compréhension orale selon la différenciation pédagogique et la jugée.

- L'élaboration d'un questionnaire pour déterminer les intérêts et les préférences des futurs enseignants autour des thèmes préférés de la compréhension orale.

- La détermination de l'intelligence multiple des futurs enseignants afin de les regrouper selon leurs IM.

2. Mesurer jusqu'à quel degré ses futurs enseignants sont dotés de ces compétences selon le pré/post test préparé par la chercheuse.

3. Préparer le modèle proposé pour développer les compétences de l'enseignement de la compréhension orale basée sur la différenciation pédagogique tout en s'appuyant sur les études théoriques concernant :

- Les modèles didactiques.

- L'enseignement de l'oral.

- la théorie de la différenciation pédagogique.

4. Mesurer l'efficacité de ce modèle sur le développement des compétences de l'enseignement de la compréhension de l'oral selon

${ }^{9} \mathrm{http}: / /$ www.differenciationpedagogique.com/questceque/questceque

\section{1}


la différenciation pédagogique ; en pratiquant le pré/post test sur les futurs enseignants de la quatrième année de la section française au Faculté de Jeunes Filles.

5. Interprétation des résultats.

6. Suggestions et recommandations.

Importance de la recherche: Cette recherche vise à:

1- Attirer l'attention des pédagogues sur l'importance de la théorie de la différenciation pédagogique dans le développement des compétences orales.

2- Attirer l'attention des pédagogues sur l'importance de la différenciation pédagogique dans l'apprentissage de la langue française.

3- Stimuler la mise en œuvre de la différenciation pédagogique au sein des futurs-enseignants afin de les pratiquer avec leurs étudiants durant le stage pratique, par suite dans leur vie professionnelle.

4- Développer et synthétiser une diversité de modèles de différenciation pédagogique et en évaluer les impacts sur la réussite des étudiants.

5- Ouvrir la voie devant les autres recherches pour développer les compétences de l'expression orale selon la théorie de la différenciation pédagogique.

6- Activer l'utilisation de l'Internet dans nos écoles (surtout les écoles expérimentales) en entraînant les enseignants à insérer des documents authentiques prises de l'Internet dans leur enseignement.

7- La nécessité de l'utilisation des multimédia dans l'enseignement des compétences orales tels que les films, la radio $\mathrm{RF} 1^{10}$, les CD-Rom etc.

VIII-Briser la barrière psychologique entre les étudiants et les multimédias en développant leurs compétences de la compréhension orale.

IX- Méthodologie de la recherche : La chercheuse a suivi la méthode quasi-expérimentale, elle a pratiqué le modèle sur 20 étudiantes puis elle a analysé les résultats statiquement.

X- Hypothèse de la recherche : Il existe une différence significative entre le total des notes du groupe expérimental au pré- post/test

${ }^{10}$ RF1: Radio France 1

\section{2}




\section{des compétences de l'enseignement de la compréhension orale en faveur du post/test}

\section{XI- Résultats de la recherche:}

La chercheuse peut avérer l'efficacité du modèle proposé pour développer les compétences de l'enseignement de la compréhension de l'oral des futurs enseignants du Faculté de Jeunes Filles selon la différenciation pédagogique elle a déterminé pendant les séquences de la recherche leurs préférences, leurs intelligences multiples, elle les a entrainées à travailler en groupes quand-même à gérer la classe pendant ce travail, elle a pu aussi développer autres que leurs compétences didactiques, leurs compétences linguistiques orales et elle a combiné l'éducation et le plaisir de l'utilisation des multimédias.

La partie théorique Cette partie de la recherche tourne autour trois axes principaux : La compréhension orale $(\mathrm{CO})$. , La différenciation pédagogie (DP). Et enfin Le modèle didactique.

Premier Axe: La compréhension orale : C'est l'aptitude la moins étudiée $^{11}$, la plus difficile et paradoxalement la plus nécessaire ${ }^{12}$. Une des raisons de l'abandon de cette compétence, comme l'a expliqué Nunan ${ }^{13}$ est qu'elle a été mise à l'écart pendant très longtemps par rapport à la production orale, elle a occupé une place secondaire dans les objectifs des personnes qui étudient une langue étrangère, pour qui écrire et parler sont la preuve de la connaissance d'une langue.

L'enseignement de l'oral a toujours fait l'objet de préoccupations pédagogiques et linguistiques chez les enseignants de français, Lizanne Lafontaine ${ }^{14}$ affirme que l'enseignement de l'oral en classe prouve qu'il peut devenir un véritable objet d'enseignement au

${ }^{11}$ Osada, N. (2004). Listening comprehension research: a brief review of the past thirty years. Dialogue Vol. 3, pp 53-66; Vandergrift, L. (2007). Recent developments in second and foreign language listening comprehension research. Language Teaching, 40, 191-210.

12 Cuq, J.-P. et Gruca, I. (2002). Cours de didactique du français langue étrangère et seconde.Grenoble : PUG.

13 Nunan, D. (2002). Listening in Language Learning. In J. C. Richards \& W. A. Renandya (Eds.), Methodology in language teaching. An anthology of current practice (238-241). Cambridge: Cambridge University Press.

${ }^{14}$ Lafontaine, L. (2004). L"enseignement -apprentissage des genres oraux à l'école secondaire québécoise. AIRDF, (p. \&é). Québec.

\section{3}


lieu de simplement demeurer un moyen de transmission du savoir. Elle révèle que les pédagogues doivent engager une rénovation de l'enseignement du français dans laquelle l'oral fonctionne par des genres déterminés.

Cornaire et al. (1998) ${ }^{15}$, abordent le thème de l'enseignement de la compréhension orale par une réflexion sur l'entraînement stratégique et son influence sur le développement de la compréhension orale. Ils révèlent que les stratégies d'apprentissage sont bénéfiques car ils estiment que, si l'on sait comment les étudiants s'y prennent pour réaliser différentes tâches d'apprentissage, il est possible de pouvoir concevoir comment les enseigner.

\section{I- Apprentissage et enseignement de l'oral :}

En demandant ce qu'on peut enseigner de l'oral, Mac McCarthy ${ }^{16}$ propose en 1988 une révolution. À partir de l'ensemble des trois P Présentation, Pratique, Production), il insiste sur l'utilisation des trois I (Illustration, Interaction, Induction).

1. Illustration : il s'agit d'exposer les étudiants à la langue réelle, avec une vidéo, un enregistrement ou le professeur parlant à la vitesse normale, (ce qui était réalisé en pratiquant le modèle proposé)

2. Interaction : Interagir ne signifie pas parler entre étudiants, ou avec le professeur, mais entendre ce que l'on voit, penser, se rendre compte de ce que le langage utilisé représente au niveau de l'apprentissage de la langue. On n'apprend pas ce qu'on ne voit pas, mais les professeurs ne comprenant pas la nécessité de changer ne changeront pas.

3. Induction : après avoir vu des exemples, les avoir intériorisés, les étudiants seront en situation de pouvoir commencer à apprendre la langue d'une façon différente.

Michèle Verdelhan-Bourgade ${ }^{17}$ affirme que «si l'on perçoit même comprendre et produire de l'oral comme le " recto-verso " d'une

15 Cornaire, C. et Germain, C. (1998) La compréhension orale. Paris : CLE International.

${ }^{16}$ L'évaluation des compétences orales en langues vivantes - Réflexions Actes du séminaire national Paris, le 23 et 24 novembre 2006 eduscol.education.fr

17 Michèle Verdelhan-Bourgade: Apprendre à comprendre l'oral en situation de français de scolarisation IUFM de Montpellier, Didaxis-Dipralang, université

\section{4}


même activité langagière, il est peut-être possible alors d'analyser la compréhension orale comme on le fait pour la production. Dégager des objectifs d'apprentissage nécessite qu'on ait aussi analysé la compréhension en ses composantes ».

On proposera donc dans notre modèle et comme l'a affirmé Michèle Verdelhan-Bourgade de considérer que, l'étudiant-maitre en cour de son apprentissage et son enseignement réalise des opérations mentales de compréhension qui sont aussi, en même temps, langagières, sociales, culturelles. Ces actes se produisent dans des situations de communication et portent sur divers objets, personnes, types de discours et voici la typologie d'actes de compréhension pratiquée dans notre modèle proposé

\section{II- Typologie d'actes de compréhension ;}

- Actes de repérage : identifier, reconnaître ; observer, écouter ; relever des indices sonores ou visuels.

- Actes d'inférence : réaliser un acte, adopter un comportement, à partir d'une demande ou d'un ordre ; déduire, conclure.

- Actes de dépassement du dit : en-decà : repérer et interpréter les implicites, les ellipses, remplir les " blancs du discours" ;

○ au-delà : anticiper, comprendre la suite ; deviner où on veut nous mener; faire des hypothèses.

- Actes de relation : associer ; aller du connu à l'inconnu, de l'inconnu au connu ; établir des relations logiques : avant/ après, cause/conséquence ; traduire ; interpréter des figures de style.

- Actes de classement : affecter à une catégorie (langue, discours, mot, référent...);trier entre fait et subjectivité; hiérarchiser les événements, les émotions; organiser le discours entendu.

- Actes de création : inventer (une suite, un discours semblable, différent...).

III- Objectifs de la compréhension orale ${ }^{18}$ : Il est question de former nos apprenants à devenir plus sûrs d'eux, plus autonomes progressivement. Notre apprenant va réinvestir ce qu'il a appris en classe et à l'extérieur, pour faire des hypothèses sur ce qu'il a écouté

Montpellier III Didactiques de l'oral les 14 et 15 juin 2002 Mis à jour le 15 avril 2011 http://eduscol.education.fr/

18 (Ducrot, 2005)

\section{5}


et compris, comme dans sa langue maternelle. Il a dans son propre système linguistique des stratégies qu'il va tester en français.

L'étudiant va se rendre compte que ses stratégies ne fonctionnent pas tout à fait et les activités de compréhension orale vont l'aider à développer de nouvelles stratégies qui vont lui être utiles dans son apprentissage de la langue. Notre apprenant sera progressivement capable de repérer des informations, de les hiérarchiser, de prendre des notes, en ayant entendu des voix différentes de celle de l'enseignant. Ce qui aidera l'étudiant à mieux comprendre les français natifs. En effet, on peut leur faire écouter des documents sonores, avec des rythmes, des intonations, des façons de parler et des accents différents. Les objectifs d'apprentissage sont d'ordre lexicaux et socioculturels, phonétiques, discursifs, morphosyntaxiques (ce qui était réalisé dans l'application du modèle proposé)

En effet, ses activités peuvent aider les étudiants à :

- découvrir du lexique en situation

- découvrir différents registres de langue en situation

- découvrir des faits de civilisation

- découvrir des accents différents

- reconnaître des sons.

- repérer des mots-clés

- comprendre globalement

- comprendre en détails

- reconnaître des structures grammaticales en contexte

- prendre des notes...

Supports audio : On doit utiliser en classe des cassettes ou des CD enregistrés, par des natifs ou des francophones, ou des documents sonores authentiques en français. ${ }^{19}$

IV- Critères de la qualité pédagogique d'un bon document sonore. Qu'on doit prendre en considération lors du choix du document : la qualité du son, la présence de bruits en arrière fond (il n'en faut pas trop non plus), la durée de l'enregistrement (ni trop court ni trop long en fonction du niveau des apprenants), le débit des

19 Annexe n:11Listes des supports utilisés par nos apprenants.

\section{6}


locuteurs (qui doit être naturel) Ce sont effectivement des critères à prendre en compte quand on décide de créer ses propres corpus et de les enregistrer.

V-Démarche didactique de la compréhension orale en classe ${ }^{20} \mathrm{~L}$ 'acte d'écouter n'est guère évident pour des apprenants. Si cet acte est banal en langue maternelle, ce n'est plus le cas en langue étrangère. Il est important de leur expliquer que le document sonore n'est pas générateur de stress en soi, qu'il est inutile de l'envisager comme un ennemi.

Après la première écoute du document : On leur demande de focaliser leur attention sur les détails de la situation en répondant simplement à des questions du type :

- Qui parle à qui ?

- Combien de personnes parlent?

- Ce sont des hommes, des femmes, des enfants ?

- Quel âge peuvent-ils avoir ?

- Où se passe la situation ?dans la rue, à la terrasse d'un café, en classe, dans une école

- Est-ce qu'il y a des bruits de fond significatifs qui aident à comprendre où ils sont? (rires, musique, bruits de rue...)

- De quoi parle-t-on?

- Quand la situation se déroule-t-elle ?

- A quel moment de la journée ou de la semaine ?

- Quel registre de langue utilise-t-on?

Ces questions sont simplement des exemples qui vont aider les apprenants, car lors de la première écoute ils auront une tâche à accomplir. Il est primordial de ne jamais leur faire écouter un document sonore sans leur dire exactement ce qu'ils ont à faire durant cette écoute. Ils doivent être actifs à chaque moment de l'écoute, pour comprendre dans un premier temps la situation, les intentions de communication, les relations des personnes entre elles.

Après la première écoute, les apprenants répondent à ces questions et feront des hypothèses grâce à ce qu'ils ont entendu. Il faut que ce soit un

${ }^{20} \mathrm{http} / / /$ www.ecoledeslangues.com

\section{7}


travail collectif, et que l'on fasse participer un maximum d'étudiants. Chaque information devra ensuite être justifiée lors de la deuxième écoute, grâce à des indices contenus dans les énoncés oraux.

Notez bien que nos étudiants-maitres ont travaillés en groupe, reparties d'après leur IM.

\section{Lors de la deuxième écoute,}

On peut leur demander de vérifier leurs hypothèses et de répondre à des questions de structuration du discours. Elles vont s'aider des articulateurs qui s'y trouvent. Les marqueurs sont des indicateurs de structuration et par exemple quand nos étudiants vont repérer le marqueur «d'abord», ils vont s'attendre à une suite chronologique avec « ensuite » ou « après »... Cette activité d'écoute active l'aidera à élucider le sens. En général, on se doit d'aider les apprenants à repérer ces mots outils, comme les connecteurs logiques (d'une part, d'autre part, ensuite...), les marqueurs chronologiques (d'abord, ensuite, puis, enfin...), les marqueurs d'opposition (mais, malgré, en dépit de, au contraire...), les marqueurs de cause et de conséquence (en effet, étant donné que ...)

La troisième et dernière écoute permettra de confirmer ou d'infirmer les hypothèses que les apprenants ont formulées ensemble.

VI- Types d'exercices en compréhension orale : Les étudiantes ont eu recours à différentes activités de compréhension et des exercices variés tels que :

- des questionnaires à choix multiples (QCM)

- des questionnaires vrai/faux/je ne sais pas

- des tableaux à compléter

- des exercices de classement

- des exercices d'appariement

- des questionnaires à réponses ouvertes et courtes (QROC)

- des questionnaires ouverts

VII- Quelques conseils didactiques en compréhension orale : La compréhension orale est probablement la première compétence traitée dès la leçon zéro. On peut utiliser aussi un document iconique comme support accompagnant le document sonore. Son rôle est de faciliter la compréhension, et non de remplacer l'explication. Elle

\section{8}


peut permettre aux apprenants d'identifier les personnages, les lieux et les aider à émettre des hypothèses concernant le contenu du dialogue avant la première écoute.

A éviter :

- Ne pas laisser les apprenants regarder la transcription du dialogue, qui se trouve généralement à la fin du manuel. Seule l'image concernant chacun des dialogues doit être présentée.

- Ne pas poser de questions exigeant une réponse trop longue, car il ne faut pas mélanger les compétences. On serait tenté de corriger l'expression orale et de demander des reformulations. Privilégions plutôt les exercices d'appariement, des questionnaires à choix multiples, des tableaux ou schémas à compléter. Evidemment, On ne doit pas évaluer l'orthographe ou la syntaxe dans les réponses aux questionnaires, car elles correspondent à un autre objectif.

- Les questions de vocabulaire/traduction.

- L'exploitation de l'image ne doit pas servir de prétexte à un recours à la traduction. Si les apprenants n'ont pas le bagage linguistique élémentaire pour répondre à des questions (pendant les premiers cours), l'enseignant dans ce cas présente seul et très rapidement la situation en français (personnages, rapport entre personnages, lieu...)

- Les questions sans aucun intérêt communicatif et qui amènent aussi à un processus de traduction implicite.

Il ne faut pas hésiter à rappeler aux apprenants qu'il s'agit de comprendre globalement. Ils n'ont pas à tout comprendre parfaitement. On peut rentrer dans le détail d'un document sonore, mais seulement en fonction du niveau réel des apprenants. On doit laisser de côté des éléments qui n'ont aucun intérêt pour leur progression dans leur apprentissage du moment.

Lorsque l'on pose des questions, il faut essayer de ne jamais trop suivre l'ordre chronologique De même, les activités de compréhension orale peuvent être un très bon moyen de commencer un cours de FLE. Elles offrent un support idéal, apportant généralement un thème, un objectif parfois grammatical inséré dans le document sonore, etc...

\section{9}


Dans le cas où une réponse d'apprenant est fausse, il est important de ne pas corriger soi-même. On peut faire réécouter une séquence du dialogue qui aide l'apprenant à se corriger lui-même. La séquence à faire réécouter doit par contre avoir un sens complet.

\section{Il est important de varier la typologie d'exercices en compréhension} orale, afin de ne pas ennuyer les apprenants. Des activités qui se présentent différemment stimulent leur esprit.

Il faut faire entendre aux étudiants l'oral public en classe de français et se pencher sur différentes variantes et contextes de diffusion des situations d'oral : discours de la radio, de la télévision, des cassettes, des disques compacts, des cédéroms et divers discours de la société.

En ce qui concerne les genres utilisés dans notre modèle proposé les étudiants maitres ont choisi des chansons, un texte explicatif, un discours de la radio et une partie d'un match de football télévisé, ce qui assure la variation dans les genres et les thèmes traités basées sur leurs IM.

VIII- L'évaluation et l'oral: Un problème se pose avec acuité : celui de l'évaluation; les activités de communication orale réalisées en classe doivent faire l'objet d'une « objectivation », pour reprendre un terme compris de tous, au même titre que les activités d'écriture, par exemple. Des moments d'évaluation formative semblent fondamentaux pour rendre les étudiants conscients de leurs forces, mais aussi de leurs nouveaux défis en apprentissage de l'oral. ${ }^{21}$

Comment évaluer l'oral: Pour l'enseignant, le point sensible de l'apprentissage de l'oral est l'évaluation. Comment juger? Sur quelles bases fixer une note? Quels critères appliquer pour évaluer?

L'évaluation de l'oral consiste à développer avec les étudiants les critères de correction qui aboutissent dans un outil d'apprentissage: les grilles d'évaluation. Ces grilles permettent à l'étudiant d'être actif, de réfléchir à sa manière de faire son travail et mène ainsi à l'autoévaluation, en passant par l'évaluation par les pairs.

21 Lafontaine, L. (2004). L"enseignement -apprentissage des genres oraux à l'école secondaire québécoise. $A I R D F$, (p. \&é). Québec.

\section{0}


Cette forme d'évaluation est indispensable si l'étudiant est censé pouvoir se corriger : l'étudiant doit aussi maîtriser les critères de réussite ou d'achèvement, il est essentiel de lui avoir donné la possibilité de s'en faire une représentation claire et cohérente. Ainsi armé, il peut prendre lui-même en charge la démarche qui lui permettra d'atteindre l'objectif visé, et l'évaluer lui-même. $)^{22}$

Toutes ces formes d'évaluation visent donc à rendre l'étudiant plus autonome et responsable de son apprentissage tout en renforçant sa motivation : " l'environnement éducatif devrai donner aux étudiants la responsabilité de leurs projets d'apprentissage. On sait bien désormais qu'ils ne parviennent à l'auto direction et l'auto motivation que lorsqu'ils acquièrent le contrôle personnel de leur éducation. $\gg{ }^{23}$

L'évaluation de l'oral comprend en fait l'évaluation des différentes compétences (l'écoute), la production orale en inter-réaction (la communication, le dialogue, les sketches) et la production orale en continu (l'exposé). Il est clair que pour chacune des compétences il y a des formes d'évaluation spécifiques.

L'évaluation comme une des étapes essentielles dans notre modèle didactique, occupe un rôle primordial, elle représente le tiers du travail, on a eu recours à l'évaluation formative tout au long de l'application du modèle, l'étudiant maitre s'évalue après chaque étape, elle réside dans l'évaluation des compétences de l'enseignement qui se sont reflétées dans l'auto-évaluation à l'aide du grille de la formulation des objectifs $^{24}$, grille de la formation des équipes ${ }^{25}$, et l'évaluation sommative finale qui réside dans le post test. ${ }^{26}$

C'est dans ce sens qu'un modèle didactique descriptif de la compréhension orale peut aider les enseignants à former leurs étudiants, car il fournit des pistes permettant de développer, entre autres, des

${ }^{22}$ DE VECCHI, Gérard, Aider les étudiants à apprendre, Hachette Livre, 1992, page 93.

23 McCOMBS, Barbara L. et POPE Ja- mes E., Motiver ses étudiants, de boeck, 2000, page 99.

${ }^{24}$ Voir Annexe n:7

${ }^{25}$ Voir Annexe n:8

${ }^{26}$ Voir Annexe n:10 
activités d'oral structurées telles que les discussions, les exposés et les débats, etc.

\section{Deuxième Axe : La différenciation pédagogique}

Dans ces lignes la chercheuse va débuter par un apport historique sur la naissance de la différenciation pédagogique, elle met l'accent sur sa finalité, ses objectifs pour, les domaines de différenciation possibles, et on termine par les démarches à suivre pour intégrer la DP dans nos classes

I- La naissance de la différenciation pédagogique: La période du concept de la pédagogie différencié a été marquée par un modèle d'enseignement-apprentissage appelé le modèle de l'empreinte : l'étudiant aurait une tête vide dans laquelle l'enseignant n'aurait qu'à faire entrer des connaissances. Dans un tel modèle, le rôle d'enseignant est essentiellement de clarifier, de simplifier le savoir à transmettre ; et celui de l'étudiant, d'être attentif et de bien mémoriser ce savoir. Petit à petit, était intégrée la pratique documentaire qui consiste à prendre appui sur des documents divers (ce qui était déjà une première approche de la différenciation) pour mettre en évidence la problématique qu'il s'agit de découvrir. Une telle pratique suscitait davantage de participation de la part des étudiants, mais pas forcément de meilleurs résultats au niveau des apprentissages.

La période suivante été marquée par la découverte d'un autre modèle d'enseignement, le modèle du conditionnement ${ }^{27}$ plus connu dans sa traduction au niveau de la profession par la pédagogie par objectifs. Il est marqué par la volonté de définir les connaissances en termes comportementalistes observables et non plus de manière mentaliste. Ce qui se traduit de deux manières dans la pratique de l'enseignant:

1. clarifier d'abord ce qu'il attend de ses étudiants au terme d'un apprentissage et comment cela s'évaluera concrètement ;

2. être transparent, explicite par rapport à ces objectifs d'apprentissage, ce qui l'amenait soit à donner une fiche des objectifs à atteindre, soit à les faire noter au démarrage d'une étude.

${ }^{27}$ (Le modèle du conditionnement se réfère aux travaux de Pavlov, Skinner, Watson) 


\section{II- Définition de la différenciation pédagogique : Louis le Grand}

désigne «C'est un effort de diversification méthodologique susceptible de répondre à la diversité des étudiants. ${ }^{28}{ }^{28}$, De Perreti ${ }^{29}$ assure que c'est une méthodologie d'enseignement et non une pédagogie. Face à des étudiants très hétérogènes, il est indispensable de mettre en œuvre une pédagogie à la fois variée, diversifiée, concertée et compréhensive. Il doit y avoir une variété de réponses au moins égale à la variété des attentes, sinon le système est élitiste. Chaque enseignant est différent dans sa manière de faire et il reconnaît à l'autre le droit d'avoir une méthode différente. La diversification est facteur de réussite. Du bon sens, de la bonne entente sont des gages de réussite. Le travail en équipe devient une obligation de service, l'enseignant ne peut rester isolé. » L. DREVILLON souligne que " La 'flexibilité méthodologique' du maître est un facteur de la réussite des étudiants dans la mesure où elle permet à chacun d'élaborer sa propre stratégie. \) 30

K Kojak ${ }^{31}$ la considère comme étant toutes les modifications dans les situations de l'enseignement permettant aux étudiants d'apprendre avec leurs collègues. Elle prétend aussi que c'est la façon de penser sur la nature de l'enseignement et de l'apprentissage et qui dépend d'une variété de stratégies permettant à l'enseignant de répondre aux besoins des différents apprenants.

Bref, c'est une attitude du formateur qui doit traiter toutes les pratiques pédagogiques mises en œuvre dans une classe pour intégrer des paramètres de différenciation se centrant sur l'organisation des situations d'apprentissage /enseignement afin de prendre en compte, au mieux, les besoins de chacun des étudiants en respectant leurs niveaux d'acquisition spécifiques et leurs rythmes d'apprentissage.

III- Pédagogie différenciée ou DP: En racontant comment et pourquoi, il est passé du concept de pédagogie différenciée à celui de

${ }^{28}$ (Louis LEGRAND1984, La différenciation pédagogique, Scarabée, CEMEA, Paris)

29 (A. De PERETTI)

30 L. DREVILLON.1980, Pratiques pédagogiques et développement de la pensée opératoire, PUF

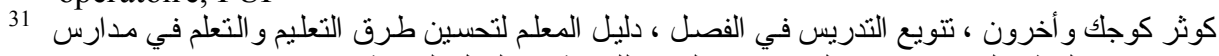

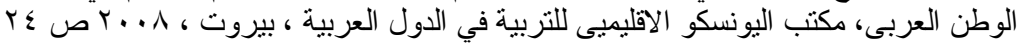


différenciation pédagogique, Hubert ${ }^{32}$ dit "je suis passé du concept de pédagogie différenciée, qui est marqué par une conception techniciste, voire simpliste, techniciste au concept de différenciation pédagogique, qui est marqué par une approche plus complexe, par un autre regard sur l'étudiant, je suis passé du pôle du « mieux enseigner » à celui du « mieux apprendre» ou autrement dit à vouloir aider les étudiants à mieux apprendre en prenant appui sur leurs différences, cela passe par une démarche qui part de l'individu et qui cherche à l'aider, à (se) construire par, avec, grâce aux autres."

Sa Finalité : est de «lutter contre l'échec scolaire tout en alliant la transmission des savoirs au développement de chaque étudiant.»33 Elle se définit donc comme étant une pédagogie de la réussite organisée en situation d'apprentissage et d'évaluation adaptées aux besoins et aux difficultés propres à chaque étudiant.

IV- Ses Objectifs : Davy EITO: ${ }^{34}$ nous présente trois objectifs fondamentaux qui constituent cette pédagogie :

1. Améliorer la relation enseignés/enseignants : Les sentiments positifs tels que le plaisir, la confiance ou la sécurité engendrent de la motivation, stimulus indispensable à tout apprentissage. La relation, affective, liant l'enseignant à l'enseigné est donc fondamentale.

La pédagogie différenciée, à travers laquelle le professeur est plus proche individuellement de ses étudiants, laisse le champ libre à l'émergence de telles émotions.

2. Enrichir l'interaction sociale : En effet, chaque étudiant placé dans un groupe peut bénéficier d'une richesse d'interaction avec ses autres camarades, ce qui lui permet de s'épanouir et d'acquérir durablement des savoirs et savoir-faire, ce qui était réalisé durant l'application du modèle.

3. Apprendre l'autonomie. Le cadre de formation de la pédagogie différenciée est un cadre souple et sécurisant, dans lequel les étudiants bénéficient d'un champ de liberté où ils ont le droit de choisir, de

${ }^{32}$ HUBERT, J.-C. (s.d.). DE LA PEDAGOGIE DIFFERENCIEE. Consulté le juin 12, 2011 sur WWW.Spirale-edu-revue.fr.

${ }^{33}$ PRZESMYCKI, Halina : La Pédagogie différenciée. Hachette Education. Paris 2011.

${ }^{34}$ Davy EITO Un essai de pédagogie différenciée: la pédagogie de contrat» Mémoire professionnel réalisé à l'IUFM de la Réunion en 1999 - 2000

\section{4}


décider, d'innover et de prendre des responsabilités. Rendus ainsi plus autonomes, les étudiants sont plus créatifs et plus imaginatifs, ce qui favorise leur développement cognitif et leur facilite les apprentissages. (ce qui était reflété dans l'application de la recherche dans les grilles d'auto-évaluation formulées et pratiquées après chaque étape de notre modèle.

V-Principes de base de la différenciation pédagogique:35_Perrenoud propose les principes suivants :

- Le développement et la remédiation des difficultés des étudiants dans leurs d'apprentissage. (Remédier les difficultés de l'apprentissage)

- Porte sur les moyens et les modalités du travail.

- Se focaliser, se centrer sur les objectifs essentiels dans une vision stratégique.

- La préoccupation de tous les méthodes, les dispositifs, les disciplines, les niveaux d'enseignement.

- Travailler en groupes, s'appuyer sur les interactions sociocognitives.

- Ne doit pas aboutir à un enseignement entièrement individualisé.

- Basée sur une observation formative, critériée, comparant chaque étudiant aux objectifs de formation plutôt qu'à ses camarades de classe.

- Engager les étudiants dans des situations-problèmes ou des projets, qui les confrontent à des obstacles, dont le dépassement devient l'objectif à court terme.

- Il ne s'agit pas d'apprendre «à son rythme », plutôt d'apprendre à un rythme relativement standard, mais soutenu de façon différenciée par les enseignants.

- Il faut différencier, c'est la part d'investissement subjectif, d'intelligence professionnelle, de créativité, d'enseignement stratégique, de prise en charge personnalisée dévolue à chaque étudiant.

- Il exige une formation pointue, en didactique, en évaluation, en métacognition, compétences sans lesquelles on ne saura ni s'écarter

35 Perrenoud: $\mathrm{Ph}$ (2005) Différencier : un aide-mémoire en quinze points » In Vivre le primaire (Québec), Presses de l'Université du Québec. N 2, mars-avril 2005, pp. 34. ». Date de pagination Décembre 2010.

\section{5}


des situations les plus conventionnelle, ni piloter les processus d'apprentissage.

- La différenciation doit être pensée et mise en œuvre en équipe, pour confronter plusieurs regards sur les étudiants, diviser le travail, gérer plusieurs groupements, travailler les objectifs et les outils ensemble.

- La DP suppose une solidarité entre étudiants et entre familles, donc leur adhésion réfléchie à l'idée de discrimination positive.

VI- Que différencier ${ }^{36}$ Dans une perspective de différenciation, les éléments pouvant en faire l'objet sont :

1- Différencier les contenus (Ce que l'étudiant apprend) : Certains étudiants peuvent apprendre, comprendre et faire certaines choses pendant que d'autres apprennent, comprennent et font d'autres choses. Les contenus se définissent par ce que les étudiants vont apprendre et le matériel qui sera utilisé à cette fin. Différencier les contenus permet de détecter les forces et les faiblesses des étudiants. Il s'agit en fait de varier et d'ajuster les tâches en fonction de l'évolution de la compétence considérée afin de s'adresser à l'étudiant à l'intérieur de sa zone proximale de développement.

2- Différencier les structures (L'environnement dans lequel se font les apprentissages et l'évaluation): L'enseignant ne pouvant être partout au même moment, il est nécessaire de pouvoir différencier les structures pour réellement faire de la différenciation pédagogique. Cette différenciation peut avoir lieu en variant des activités en groupe, en sous-groupes et individuelles, tout en facilitant le travail d'entraide en formant les sous-groupes selon différents modes (selon les besoins et l'IM dans notre application du modèle).

3- Différencier les processus (Les moyens avec lesquels se font les apprentissages et l'évaluation): Il s'agit de varier les moyens et les situations d'apprentissage en tenant compte des

${ }^{36}$ Source : Ministère de l'Éducation, du Loisir et du Sport. La différenciation pédagogique : théorie et applications. Groupe de travail sur la différenciation pédagogique en Outaouais. Synthèse des travaux réalisés dans le contexte des priorités du Comité régional de coordination pédagogique ( $\mathrm{CRCP})$. Bibliothèque nationale du Québec, 2005, pages 24-28.

\section{6}


diverses manières dont les étudiants traitent l'information pour favoriser l'apprentissage visé. Pour se faire, on peut varier les approches pédagogiques : enseignement stratégique, enseignement par médiation, apprentissage coopératif, pédagogie par projet, etc.

\section{4- Différencier les productions (Comment l'étudiant montre ce}

qu'il a appris) : C'est permettre aux étudiants d'utiliser des véhicules différents pour montrer ce qu'ils ont appris ou compris $\mathrm{du}$ contenu. La différenciation des productions nous amène à parler de l'évaluation. Dans notre modèle, enseignement et évaluation sont indissociables et se poursuivent dans la régulation.

Bref, ces éléments sont généralement inter reliés et dans tous les cas impliquent une variation dans le soutien accordé aux étudiants. On peut difficilement intervenir par rapport à un d'entre eux sans toucher aux autres. L'application de la différenciation en classe peut prendre différentes formes et intensités. Le degré de différenciation dans une classe se fera en fonction des besoins des étudiants, de la connaissance qu'en a l'enseignant, des possibilités qu'il a de tenir compte des différences et de sa capacité à mettre en place des structures différenciées. Enfin, la DP implique le partage des responsabilités avec les divers intervenants scolaires.

VII- Démarches de la différenciation pédagogique: Pour mettre en œuvre la différenciation pédagogique, nous adoptons la démarche suivante en cinq étapes, proposée par Marie-Hélène Guay ${ }^{37}$ :

Étape 1 : Définition de la situation actuelle et d'une problématique (évaluation diagnostique) : une analyse de la situation actuelle et de la problématique relative à l'apprentissage des étudiants doit être effectuée. Essentiellement, il s'agit de faire l'évaluation diagnostique des composantes et relations de la situation pédagogique. Dans cette recherche c'est le cas des futurs enseignants qui souffrent d'un manque des ateliers qui tendent à développer leurs compétences d'enseignement et d'apprentissage surtout dans les compétences orales. La chercheuse

${ }^{37}$ Chercheuse en éducation et conseillère pédagogique de la Montérégie au Québec (Canada) à la Commission scolaire des Trois-Lacs (Vaudreuil-Dorion). Recherche subventionnée par la Coopérative de développement régionale de la Montérégie décembre 2007

\section{7}


aussi pourra se poser les questions suivantes : "Qu'est-ce je connais des attitudes, des savoirs, des démarches d'apprentissage ou des processus métacognitifs de mes étudiantes ? Est-ce que mes interventions pédagogiques sont adéquates pour les aider ? Sont-elles adaptées à leurs préalables et caractéristiques ? Les supports visuels présentés sont-ils pertinents pour eux ? Ont-ils eu suffisamment de temps pour apprendre? Effectuée à partir de telles questions, une évaluation diagnostique vise une meilleure compréhension de ce qui entrave l'apprentissage.

Étape 2 : Définition de la situation désirée : Une fois la situation actuelle bien définie, il est possible de préciser la situation désirée, c'està-dire ce que les étudiantes doivent apprendre et ce qui sera amorcé ou modifié, au sein de la situation pédagogique, pour y contribuer. Une diversité d'actions, d'outils ou de types d'interventions peuvent alors être envisagés : sélectionner certains contenus, modifier les formules pédagogiques, créer un matériel ou un support visuel, ajuster le programme d'activités, varier les modes de regroupement, etc. Par exemple, la chercheuse ayant constaté que ses étudiantes ne maîtrisent pas les stratégies d'enseignement des compétences orales parce qu'elles ne les enseignent pas explicitement, pourrait juger désirable d'accentuer l'enseignement explicite de telles stratégies pour que celles-ci soient capables de les utiliser adéquatement.

Étape 3 : Planification de l'action: La planification de l'action correspond au moment où un enseignant conçoit et se prépare à mettre en œuvre les modifications jugées désirables à la situation pédagogique. Dans notre cas, cela pourrait correspondre à des lectures sur les compétences nécessaires pour l'enseignement de l'orale selon la DP, la préparation des leçons: la formulation des objectifs ; la formation des groupes et des sous-groupes, la théorie de l'IM et enfin l'évaluation formative et sommative.

Étape 4 : Action : L'action correspond à la mise en œuvre effective des actions planifiées permettant de passer de la situation actuelle à la situation désirée. Dans l'exemple retenu, l'action correspondait au moment où la chercheuse entrainerait les futurs enseignants au modèle proposé afin de développer leurs compétences de l'enseignement de l'oral tout en réalisant la différenciation pédagogique. 


\section{Étape 5 :}

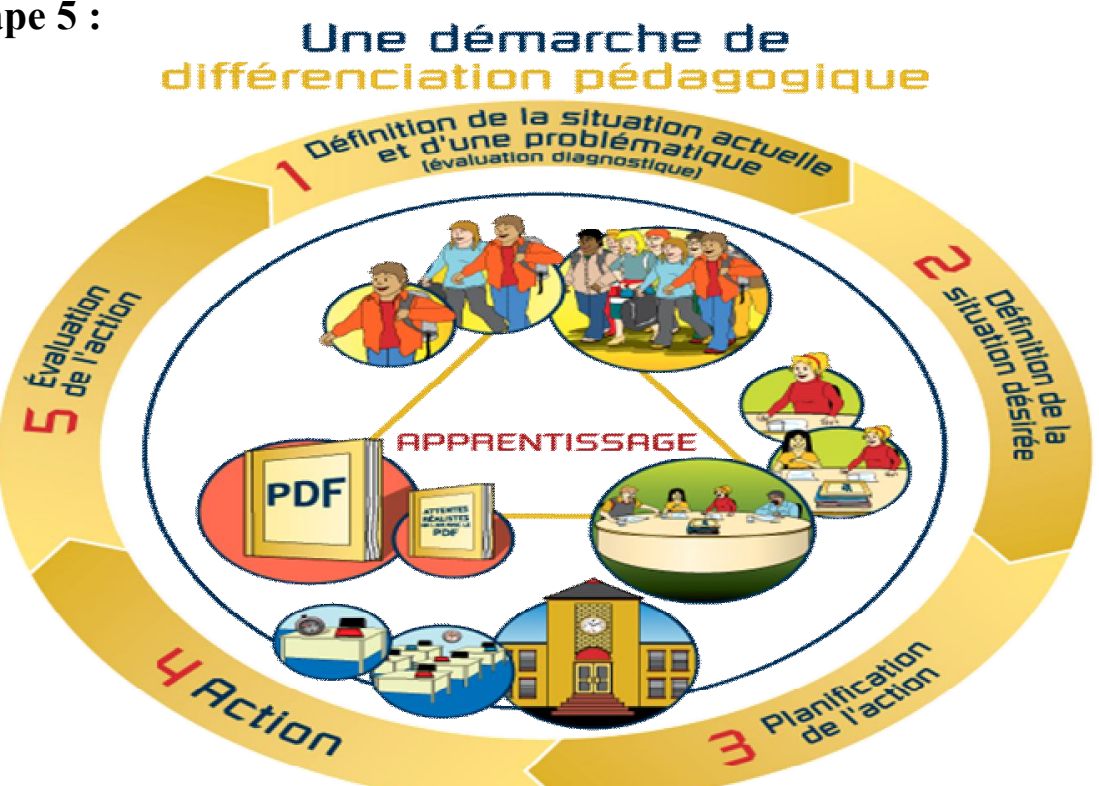

Évaluation de l'action : Au terme de toute démarche de différenciation pédagogique, il y a lieu d'évaluer l'impact des actions mises en œuvre sur l'apprentissage des étudiants. Toujours en lien avec notre exemple relatif aux difficultés d'un sous-groupe d'étudiants en lecture, l'étape d'évaluation de l'action pourrait correspondre au moment où la chercheuse, à la fin du terme, pratiquait le post test pour évaluer leur degré de maitrise des compétences de l'enseignement de l'oral selon le modèle proposé basé sur la DP.

Bref, on peut constater que pour bien préparer les pédagogues à différencier dans leur classe, il nous apert nécessaire d'être ouvert sur la différence. La DP implique un changement de paradigme allant de l'enseignement centré sur la matière à l'enseignement centré sur l'étudiant ; d'avoir, une bonne connaissance des caractéristiques d'un ou plusieurs étudiants (portrait de classe, portrait des capacités et des besoins, l'IM, les styles d'apprentissage, et enfin d'avoir une bonne maîtrise des programmes de formation de nos Facultés de Pédagogie.

\section{Troisième Axe: Le modèle: définition du concept:}

Dans les lignes à venir la chercheuse va recenser quelques modèles traitant l'enseignement de l'oral ; l'étude de ces modèles lui a permis de tracer un portrait plus clair de certains éléments essentiels à l'élaboration

\section{9}


d'un modèle didactique de l'enseignement des compétences orales basé sur la DP.

I- Le « modèle didactique »: Il nous parait importante de présenter quelques définitions du ce concept, (Walliser, 1977 ; Bertrand, 1979; Gosselin, 1990 ; L'Écuyer, 1990 ; Poisson, 1990 ; Sauvé, 1992; Legendre, 1993) tous ces pédagogues affirme qu'un modèle est un système qui simplifie la réalité et qui représente une synthèse de l'objet à l'étude, en l'occurrence l'enseignement de l'oral comme il est vécu par certains enseignants de français langue première du secondaire.

Sauvé $^{38}$ affirme que le modèle didactique est donc une représentation d'un certain type de processus d'organisation et de gestion de la situation pédagogique, en fonction de buts ou objectifs globaux particuliers et en relation avec un cadre théorique qui le justifie et lui confère une dimension exemplaire, prescriptive. Rey Alain ${ }^{39}$ signale: «c'est ce qui sert ou doit servir d'objet d'imitation pour faire ou reproduire quelque chose $»$, Hegazy $^{40}$ a réclamé que c'est le plan qui a pour but d'orienter le travail d'un enseignant en classe pour faciliter sa tâche, en présentant les composantes de ce plan et les relations entre elles.

Bref, on peut constater qu'un modèle didactique est une simplification de la réalité et une synthèse de l'objet d'étude en l'occurrence celle de l'enseignement de la compréhension orale des étudiantes-maitres de la faculté des Jeunes Filles, cette simplification permet une meilleure compréhension de l'objet. ${ }^{41}$

On peut signaler aussi que dans notre recherche, le modèle proposé représente toutes les étapes à suivre par le futur enseignant pour différencier son enseignement de l'oral.

38 (Sauvé, 1992, p. 183).

${ }^{39}$ Rey Alanin et autres1986. Micro Robert. Dictionnaire de français. Montréal.

Canada. P675

${ }^{40}$ Khaled Muhammad El Hegazy 2004: Efficacité d'un modèle d'enseignement proposée en vue de développer quelques compétences de la pensée critique chez les étudiants de section française à la faculté de Pédagogie. Université de Hélouân.

41 Gosselin, P. (1990). Vers un modèle de modélisation. Dans L'association internationale de pédagogue expérimentale de langue française section canadienne (Dir.), Les modèles en éducation (p298-304). Montréal : Edition Noir et Blanc.

\section{0}




\section{II- Différence entre «Une méthode d'enseignement» et « un} modèle pédagogique :

Une méthode d'enseignement, c'est un ensemble de choses à faire ${ }^{42}$. Elle se situe toujours au niveau du "comment", sans avoir besoin d'expliciter de façon approfondie le "pourquoi". Pour être très juste, disons que, dans une méthode, les objectifs à atteindre sont supposés connus et évidents. Un modèle pédagogique, c'est un ensemble de mises en relation entre le "pourquoi" et le "comment", le "pourquoi" étant défini, d'une part, à partir des travaux de recherches dans les domaines qui concernent la pédagogie : les contenus à enseigner, la psychologie des enfants et des adolescents, et le fonctionnement de l'acte d'apprendre, d'autre part, en fonction d'une philosophie de l'homme et d'un type de Société considéré comme souhaitable. Si bien qu'un modèle pédagogique se présente, non comme un ensemble de directives, mais comme une suite de raisonnements de type "si on veut obtenir tel résultat alors, les recherches actuelles proposent de travailler de telle manière...".

Un modèle pédagogique, c'est un système. Et comme tout système, les éléments qui le constituent ne peuvent être modifiés sans modifier tout le système, voire le détruire.

\section{III- Le Modèle de Dolz et Schneuwly (1998)43:}

Il est indispensable de recenser quelques modèles utilisés en enseignement des langues, parmi ces modèles on a bien profité de ceux élaborés par Dolz et Schneuwly 1998, ce modèle rende compte de l'enseignement des genres de l'oral, il propose des séquences didactiques élaborées sur divers genres, ils suivent toutes la même démarche, mais diffèrent dans le contenu : production initiale, état des connaissances des étudiants et de leurs représentations, réalisation d'un projet concret réalisé pour quelqu'un qui est déclencheur dans la production initiale, réalisation de productions simplifiées pour enrichir la production finale ce modèle didactique répond à certains critères de validité, il possède aussi une cohérence interne et un clarté remarquable.

42 Eveline, Pour ne pas confondre un "modèle pédagogique" avec une "méthode d'enseignement". Education, Ecole et Pédagogie 2008

http://www.charmeux.fr/blog/index.php

${ }^{43}$ Lafontaine, L. (2004). L'enseignement -apprentissage des genres oraux à l'école secondaire québécoise. AIRDF, (p. \&é). Québec. Actes du $9^{\mathrm{e}}$ colloque de l'AIRDF, Québec, 26 au 28 aout. P4

\section{1}




\section{IV-Les 5 axes autour desquels se concentrent les ateliers de}

\section{l'exécution du modèle :}

1. Modélisation totale par l'enseignant ou par l'étudiant : modélisation suivie d'un retour en grand groupe afin d'identifier les forces et faiblesses concernant le genre étudié.

2. Apprentissage des rôles à jouer.

3. Apprentissage reliés aux types de pratiques : par exemple, connaitre les règles et le fonctionnement du débat: discerner le débat parlementaire du débat régulé :

4. Apprentissage des techniques d'écoute : encore peu travailler en classe de français, les maitres travaillent l'écoute à travers la reformulation et la discussion.

Lorsque la série d'ateliers formatifs est terminée, il va sans dire que les étudiantes sont prêts à être évaluées de façon sommative dans leur production finale, l'impact sur les apprenants est donc positif, ils ont appris à prendre en charge leur propre communication orale et ont développé des compétences durables et transférables a d'autre situations scolaires et quotidiennes ${ }^{44}$.

\section{Les modèles de l'enseignement de l'oral commentés par Tochon (1997): :}

Le modèle de Tochon (1997) propose d'utiliser la prise de parole afin de renforcer les corrélations, dans un projet commun, entre les manifestations du langage. Il souhaite faire réaliser en classe des projets d'intégration de la lecture, de l'écriture et de l'oral afin qu'un même sujet puisse toucher ces trois éléments de la langue. Il faut cependant être conscient de la longueur et de l'exigence que demande un tel projet pour être réalisé.

Les modèles intégrés de l'enseignement de l'oral commentés par Tochon (1997) montrent qu'il se développe à partir des diverses matières enseignées à l'école. Dans cette perspective, l'oral est intégré au contenu du cours.

Tochon (1997) suggère un tel modèle qui unit les relations entre les domaines du français (orthographe, grammaire, conjugaison,

44 Lafontaine, L. (2004). L'enseignement-apprentissage des genres oraux à l'école secondaire québécoise. $A I R D F$, (p. \&é). Québec. Actes du $9^{\text {e }}$ colloque de l'AIRDF, Québec, 26 au 28 aout 2004. P4

${ }^{45}$ Lizanne La Fontaine .opcit. 
vocabulaire) dans des projets ou des expériences, sans pour cela négliger les apprentissages oraux particuliers.

Conclusion : Nous estimons que cette recherche apporte un élément neuf à la didactique de l'oral, car la chercheuse a élaboré de façon beaucoup plus détaillée l'oral utilisé comme un objet d'enseignement, entre autres en le reliant aux activités d'oral planifiées et spontanées. Elle a également pu constater que les ateliers formatifs à l'oral se divisaient en cinq types bien précis : la modélisation totale par l'enseignant et parfois par l'étudiant, l'apprentissage des rôles à jouer, l'apprentissage lié aux types de pratiques, l'apprentissage relié aux faits de langue et l'apprentissage des techniques d'écoute.

Il est également clair que cette étude a dressé un portrait d'une réalité qui a probablement déjà changé. On rappelle qu'un modèle est imparfait, car il représente certains éléments de la réalité au détriment d'autres. Finalement, à l'aube de changements didactiques dans l'enseignement universitaire, on pense que la "didactisation » de ce modèle par les formateurs, les conseillers pédagogiques et les enseignants pourrait s'étendre à d'autres disciplines telles que l'enseignement secondaire, l'univers social.

Finalement, on a dégagé les modèles implicites de l'enseignement de l'oral des sujets de cette étude qu'on a mis en relation avec les composantes de modèle théorique d'enseignement des langues issues du cadre théorique.

\section{Cadre pratique : Introduction:}

Après ce panorama d'étude théorique dans laquelle la chercheuse a abordé les principes de base du modèle proposé, on présente dans les lignes à venir la partie pratique contenant (les outils, les étapes de l'élaboration, l'application et l'évaluation du modèle proposé, l'échantillon ainsi que sa description) ce qui répond à la troisième question de la recherche

Les outils élaborés par la chercheuse sont présentés en ordre d'exécution:

1- Liste des étapes à suivre pour enseigner la compréhension orale selon la DP.

2- Test pour déterminer les IM des étudiants -maîtres.

3- Grille concernant les critères de choix d'un document sonore et audiovisuel.

\section{3}


4- Fiche d'auto-évaluation pour la formulation des objectifs opératoires.

5- Grille concernant les critères à suivre pour la formation des équipes selon la DP.

6- Listes des sujets traités durant le travail initiatif afin de préparer les étudiantes-maitres à l'adaptation du modèle proposé.

7- Le modèle proposé.

8- Le pré/post test élaboré pour déterminer jusqu'à quel point les étudiants-maîtres ont possédé les compétences de l'enseignement de la CO selon la DP.

9- Fiche d'exploitation d'un document (écrit, sonore, visuel)

Echantillon de la recherche: L'échantillon de la recherche est au nombre de 23 étudiantes de la 4ème année du département de la langue française de la Faculté de Jeunes Filles, Université d'Ain Shams, ces étudiantes qui sont issues des écoles de langue souffrent d'un niveau très bas surtout sur le niveau des compétences orales. 4 parmi elles se sont absentées durant l'exécution de la recherche, ce qui a mené la chercheuse à se contenter de 19 maitres étudiantes qui ont continué l'expérience et ont passé par le post test.

Application de la recherche: cette recherche a eu lieu au premier terme de l'année scolaire 2011/ 2012, l'application du modèle a pris près de 3 mois, deux fois par semaine, chaque fois 2 heures, nombre totale 48 heures de travailles, l'exécution de la recherche s'est passé dans la salle de conférences en utilisant l'ordinateur portable de la chercheuse et 3 autres ordinateurs portables appartenant aux étudiantesmaitres.

$\underline{I}$-Liste concernant les étapes à suivre pour enseigner la compétence de la compréhension orale selon la DP :46

But : Le but de cette liste est de préciser les étapes que chaque étudiante maitre va suivre pour réaliser la différenciation pédagogique dans son enseignement pour développer les compétences de la compréhension orale, par suite la première question de la recherche s'est réalisée.

Sources : Pour élaborer cette liste la chercheuse a étudié ce qui suit :

- Les stratégies concernant l'enseignement de la compréhension orale.

${ }^{46}$ Voir Annexe n : 1

\section{4}


- Les compétences didactiques nécessaires à l'enseignement de la compréhension orale selon la DP.

- Les principes de base de la DP.

- Le rôle de l'enseignant du FLE selon la DP.

- Le rôle de l'apprenant du FLE selon la DP.

- Le descriptif de quelques modèles visant le développement des compétences de l'enseignement du FLE.

- Les compétences essentielles de l'enseignement: la planification, l'exécution, et l'évaluation.

Description : La liste se compose de 18 compétences qui portent sur des savoirs et des savoir-faire didactiques. Après la passation de cette liste au jury ${ }^{47}$ ces compétences étaient réduites en 8 compétences.

Cette liste est utilisée comme guide pour les étudiantes-maitres pour leur aider durant l'exécution de la recherche afin d'arriver à l'application du modèle proposé, elle sert aussi comme outil d'évaluation, afin d'être tout le temps sur le bon chemin.

\section{II- Test pour déterminer les IM des étudiantes-maîtres ${ }^{48}$.}

But: Ce test a pour but de déterminer les IM et son degré de disponibilité chez les étudiantes-maîtres à la faculté de jeunes filles, pour aider la chercheuse à varier les documents choisis, à choisir les exercices adéquates à leurs IM, afin de réaliser les objectifs de la recherche et de leur entraîner à pratiquer ce test avec leurs étudiants et de pouvoir varier leurs enseignements de la compréhension orale.

Sources: La chercheuse a adopté le test préparé par Raymond le blanc ${ }^{49}$ qui a paru dans «éducation et francophonie » 2000.C'était une version simple, clair, adaptée avec le niveau des étudiants-maîtres

Description: Sous sa forme initiale, le test se compose de 80 phrases réparties sur les différents type d'intelligences chacun comprend 10 phrases.

\footnotetext{
${ }^{47}$ Voir Annexe n:2

${ }^{48}$ Voir annexe n: 3

49 Raymond Le Blanc, in « éducation et francophonie » vol. xxv, no 2. Pour le volet I du programme de sensibilisation aux nouvelles approches pédagogiques de la commission scolaire de la rivière du Nord. France 2001.
}

\section{5}


Résultats du questionnaire: ${ }^{50}$ Après l'application du test des IM, la chercheuse peut signaler que l'intelligence la plus commune entre les étudiantes, était l'intelligence Intra personnelle dont douze parmi les vingt étudiantes possèdent cette intelligence intra personnelle Tandis que les taux d'autres intelligences étaient comme suit : 8 étudiantes jouissent de l'intelligence interpersonnelle, 7 de l'intelligence musicale, 5 de l'intelligence linguistique et le même nombre pour l'intelligence spatiale, ce qui a mené les étudiantes-maîtres à choisir les documents enseignées selon ces variations.

Selon les résultats du questionnaire, les étudiantes-maitres ont été réparties en 3 groupes reflétant les 3 IM (Interpersonnelle, musicale et linguistique).

\section{III- Grille concernant les critères de choix d'un document sonore et audio-visuel. ${ }^{51}$}

But : choisir le document sonore/audiovisuel utilisé par l'étudiantemaitre selon des critères bien déterminés, et aussi s'autoévaluer durant la phase de planification du modèle proposé.

Source : grille élaborée par la chercheuse durant l'exécution d'une recherche intitulée «Former les enseignants du FLE à didactiser les documents médias selon la pédagogie de la tâche» ${ }^{52}$

Description: la chercheuse a précisé 8 critères que l'étudiante-maitre doit suivre en choisissant les documents audio-visuels.

\section{IV- Fiche d'auto-évaluation pour la formulation des objectifs opératoires: 53}

But : Formuler les objectifs opératoires selon des critères déterminés, et s'autoévaluer en formulant les objectifs concernant les documents sujets de l'enseignement de la compréhension orale

Source : élaborée par la chercheuse.

Description : Cette fiche se compose de 9 phrases qui reflétant les étapes à suivre durant la formulation des objectifs, aussi elle représente l'auto-évaluation de l'étudiant-maitres durant la phase de planification.

\footnotetext{
${ }^{50}$ Voir annexe 5

${ }^{51}$ Voir annexe n:6

52 Sherine Darwiche et Nesrine Salah Recherche publiée dans la revue de l'association des programmes Janvier 2011

${ }^{53}$ Voir annexe n: 7
} 
V- Grille concernant les étapes à suivre pour la formation des équipes selon la DP.54

But : Formuler les équipes du travail selon des critères déterminés et s'autoévaluer tout en exécutant le modèle proposé.

Source: élaborée par la chercheuse.

Description : se compose de 8 étapes guidant les étudiantes-maitres durant leur travail en groupe, et en même temps sert comme référence pour eux durant leurs stage pratique lors de leurs applications de l'apprentissage coopératif, ainsi dans leur champs professionnels.

VI- VI Listes des sujets traités durant le travail initiatif afin de préparer les étudiantes-maitres à l'adaptation du modèle proposé55.

But: Avant l'application du modèle proposé une étude théorique des principes de base de la philosophie des DP semble être nécessaire, ce qui a mené la chercheuse à l'étudier à profond avec eux.

Source: les théories et les recherches concernant la DP, l'IM et l'enseignement de la $\mathrm{CO}$.

Description : Voici les objectifs spécifiques de l'étude concernant les thèmes étudiés avec les étudiants maitres avant l'application du modèle proposé:

- Définir les concepts de la DP, l'IM et la CO.

- Déterminer les principes de la pédagogie de la DP

- Distinguer entre les IM différentes.

- Inférer les conséquences pédagogiques de la théorie de l'IM

- Enseigner la compétence de la compréhension orale selon la DP.

- Différencier les moyens et les outils de l'évaluation en enseignant les compétences de la $\mathrm{CO}$.

${ }^{55}$ Voir annexe : 9

\section{7}


- Préparer une fiche d'exploitation pour travailler un document sonore, vidéo ou écrite afin d'enseigner la compétence de la $\mathrm{CO}^{56}$

- Adopter le modèle proposé pour enseigner la compréhension orale

\section{VII- Le pré/post test.57}

But: A la lueur des compétences déjà déterminées, la chercheuse a élaboré un test dont l'objectif est d'évaluer les compétences des étudiantes-maitres du FLE à enseigner la $\mathrm{CO}$ selon la DP. (Ce qui répond à la deuxième question de la recherche. Le même test sera postérieurement appliqué après la mise en application du modèle proposé afin de mesurer son efficacité à développer les compétences des étudiantes-maitres du FLE à enseigner les compétences de la $\mathrm{CO}$ selon la DP. (ce qu'a demandé la chercheuse dans la dernière question de la recherche)

Source : préparé par la chercheuse.

Description : Ce test se compose de deux parties : une partie théorique reposant sur les connaissances concernant les concepts de base des théories déjà étudiées telles que la DP, l'IM et l'enseignement de la CO, elle se forme des QCM, des exercices d'association, et juste et faux, et la deuxième partie pratique, l'étudiante essaye de pratiquer le modèle déjà acquis durant les cours pour enseigner la $\mathrm{CO}$ selon un document (audio- vidéo, ou écrit) selon le thème déjà choisi et aussi selon son IM déjà déterminer par le test de l'IM.

Le calcul du temps: Etant donné que le pré/post-test est composé de deux parties, nous avons jugé nécessaire de consacrer aux questions appartenant à chaque partie un temps fixe pour y répondre.

\section{A la lueur de cette formule,}

Temps moyen $=$

nps mis par la lère atudiante-maitre+temps mis par la dermiere

$\frac{+30}{2}=20$ minutes pour la première partie.

${ }^{56}$ Voir annexe n: 12

${ }^{57}$ Voir annexe n: 10 
$\frac{\text { nps mis par } 1 \text { è etudiante-maitre +temps mis par la dermiere }}{+60}=$

$\frac{+60}{2}=40$ minutes pour la deuxième partie

-le temps attribué à la première partie est 20 minutes --le temps attribué à la deuxième partie est 40 minutes.

-Ainsi le temps du test dans sa totalité est 60 minutes (une heure)

Fidélité: Pour mesurer la fidélité du pré post /test nous avons eu recours à une application du pré post/test suivie d'une deuxième application, la comparaison entre les deux passations était mesurée par une équation qui a assuré la fidélité du pré- post test.

Validité: Une autre équation concernant la validité a été appliquée, elle a assuré à son tour la validité du pré post test.

\section{VIII - Le modèle proposé en $^{58}$}

En proposant ce modèle aux étudiantes-maîtres de Faculté de jeune fille la chercheuse vise à les y entrainer en guise de l'adopter eux même avec leurs étudiants dans leur classe du FLE.

Les principes de base de ce modèle sont: La théorie de la DP, les intelligences multiples et leurs implications pédagogiques, les genres de l'enseignement de l'oral, les types des documents oraux et les techniques de travail appropriées, et les démarches à suivre pour enseigner la compréhension orale.

But : L'objectif général du modèle est de développer les connaissances, les habilités et les attitudes des étudiantes-maitres à enseigner la $\mathrm{CO}$ selon la pédagogie de la DP. Alors la troisième question de la recherche s'est exécutée.

Source : élaboré par la chercheuse.

Objectif général : Enseigner la compétence de la $\mathrm{CO}$ selon la pédagogie de la DP

\section{Objectifs spécifiques :}

- Inférer les principes de base de la théorie de la DP.

- Définir les intelligences multiples.

${ }^{58}$ Voir annexe: 11

\section{9}


- Déterminer les implications pédagogiques de la théorie de l'IM.

- Différencier entre les genres de l'enseignement de l'oral

- Distinguer entre les types des documents oraux.

- Suivre les techniques de travail appropriées à chaque document.

- Formuler les objectifs opérationnels pour chaque type de document

- Gérer à bon escient le groupe de travail en classe.

- Elaborer des exercices appropriés aux niveaux des étudiants.

- Déterminer les principes de bases de la variation de l'évaluation.

- Pratiquer le modèle de l'enseignement de la compréhension orale dans leurs classes de FLE.

Le déroulement du modèle: Afin d'appliquer le modèle proposé, la chercheuse discute avec les étudiantes-maîtres les principaux axes déjà cités $^{59}$, les étudiantes-maitres sont repartie en 4 groupes selon leurs IM : groupe de l'Intelligence Interpersonnelle (6 étudiantes-maitres), l'Intra personnelle (5), Musicale(4), et Linguistique (4) Ce modèle d'enseignement s'appuie sur les trois axes présentant les compétences générales de l'enseignement: La planification, l'exécution et l'évaluation.

1. Planification: C'est une phase de préparation qui aura lieu la veille de la leçon Dans cette phase chaque groupe va réaliser, les étapes suivantes.

1. Choisir le document déclencheur (écrit, sonore ou vidéo) pour enseigner la compréhension orale, selon des critères déterminés.

2. Faire la transcription du document.(si c'est un document audiovisuel)

3. Formuler des objectifs opératoires de chaque document.

4. Préparer un des trois types de document oral: (écrit, sonore, audio-visuel) en respectant les techniques d'exploitation appropriées.

2. Exécution: Ce qui se déroule en classe lors de l'exécution du modèle proposé:

1. Identifier les IM des étudiantes en élaborant le test de l'IM.

2. Trier les étudiantes selon leurs intelligences multiples.

${ }^{59}$ Voir annexe: 9

\section{0}


3. Former des groupes des étudiantes en se basant sur leurs IM, leurs intérêts ou leurs thèmes préférés.

4. Exploiter chaque type de document oral : écrit, sonore, audiovisuel en suivant les techniques d'exploitation nécessaires.

Evaluation : L'évaluation est sommative, elle se fait individuellement et par groupe, elle commence avec la préparation qui aura lieu la veille de la leçon jusqu'à la fin de la classe. Elle réside dans l'auto-évaluation immédiate de chaque étape réalisée.

\section{S'évaluer durant la phase de la planification :}

Cette phase d'évaluation sera individuelle chaque apprenant s'auto évalue après la préparation de son travail

1- Grille pour évaluer son choix du document déclencheur afin d'enseigner la compétence orale.

2- Grille pour évaluer le choix du document vidéo.

3- Grille pour s'assurer de la formulation correcte des objectifs opératoires

\section{$\underline{\text { S'évaluer durant la phase de l'exécution }}$}

Cette partie représente une coévolution, chaque groupe évalue soimême, et aussi évalue les groupes collègues

1- Grille d'auto-évaluation afin d'évaluer la formation des groupes et la répartition du travail dans chaque groupe.

Cette phase aura lieu par l'enseignant et aussi par les autres groupes, une discussion aura lieu après la présentation de chaque groupe afin de préciser les points forts et les points faibles de chacun, les membres de groupe commencent à faire un commentaire sur soimême par suite les autres groupes donnent leurs avis et finalement l'enseignant résume et donne la conclusion.

\section{VIII- Fiche d'exploitation du document support :}

But : L'objectif de cette fiche est de sensibiliser les étudiants maitres à exploiter les documents supports de l'enseignement de la compétence de la $\mathrm{CO}$, tout en élaborant cette fiche selon les types variés du document déclencheur utilisé...

Source : élaboré par la chercheuse. 
Description : cette fiche représente la préparation écrite du cours enseigné dans laquelle l'étudiante doit préciser l'objectif général de l'enseignement du document, les objectifs, opérationnels, les objectifs linguistique, communicatifs, acte de parole et aussi les genres des exercices. En utilisant un document vidéo, elles doivent préciser le nombre et les objectifs de chaque visionnement en précisant les activités demandées des étudiantes à la suite de chaque phase. ${ }^{60}$

\section{Où se situe la philosophie de la DP dans ce modèle :}

La philosophie de la DP réside dans ce modèle en ce qui suit :

- Enseigner la $\mathrm{CO}$ selon des sujets différents liés à la compétence à développer et basés sur les préférences des apprenants

- Travail individuel, en dyade, en équipe, collectif (selon les exigences de la situation)

- Varier les regroupements d'étudiants (besoins, niveaux, intérêts, préférences approches, démarches, projets, etc.)

- Varier les rôles dans chaque groupe afin que chaque membre dans le groupe joue tous les rôles proposés.

- Varier les documents déclencheurs (écrit, sonore et audio-visuel)

- Varier les types des documents écrits : lettre d'amour, page d'un magazine de mode, article sanitaire sur les dangers de cellulaire, recette de cuisine)

- Varier les types de chaque document sonore (météo, une interview avec un acteur célèbre, un feuilleton d'une célèbre série)

- Varier les types de document vidéo (une émission qui couvre le match de football féminin, un clip de Céline Déon, un discours comique de la femme de Jacques Chirac)

- Varier le niveau auquel on prépare les leçons (débutant et niveau A2)

- Horaires variés : accorder plus de temps si la tâche est plus difficile ou plus longue que prévue.

- Utilisation de coins ateliers, place privilégiée dans la classe (près de l'enseignant, loin de sources de distraction, etc.)

${ }^{60}$ Voir Annexe n: 12

\section{2}


- Types de consignes différentes : collectives, individuelles, fermées, ouvertes, reformulation, rappel

- Variété d'outils : matériel de manipulation, ordinateur, référentiels visuels

- Utilisation de diverses approches et théories pédagogiques : intelligences multiples, enseignement stratégique, modélisation, pratique guidée, pratique autonome.

- Différentes procédures d'évaluer : Co évaluation, entrevue, observation directe

- Démarches variées s'ajustant aux intelligences multiples.

- Développer, avec les étudiants, un coffre à outils support : radio vidéo, film, chansons, recueil de concepts, liste de vérification, sites internet.

- Varier les média de présentation différents : ordinateur, magazines, journaux enregistrement audio, CD.

- Types d'évaluations différentes : fiches ou grilles d'autoévaluation et de Co évaluation, évaluation collective orale.

\section{Commentaire général sur l'application du modèle :}

On peut constater que les étudiantes, dans la grande majorité, se sont bien impliquées en pratiquant la DP et que les plus faibles notamment y ont vu l'opportunité de réaliser une tâche à leur portée, où ils pouvaient mettre en valeur des compétences de savoir-faire plus que de savoir.

- L'idée de réunir une étudiante faible avec une étudiante plus fort a permis à tous les groupes de rendre leurs travails plus que corrects car le savoir des un allié au savoir-faire des autres a porté ses fruits.

- L'idée d'intégré la DP durant les séquences pratiques de notre recherche a permis à quasiment toute la classe d'accrocher sur le sujet et de poursuivre l'apprentissage sur de meilleures bases.

- En adoptant la DP, elle a permis à chaque étudiante d'avoir la possibilité de suivre le cours à son niveau. En effet, les étudiantes qui étaient incapables de faire une activité où il fallait répondre par des phrases complètes par exemple, peuvent désormais faire aussi cette activité en répondant à l'aide d'un QCM. Chacun peut suivre selon son niveau mais aussi selon son rythme car les fiches préconçues permettent aux plus rapides de s'avancer par rapport aux autres sans que cela ne lui pose de problème pour leur trouver une activité.

Interprétation des résultats : Apres avoir examiné les outils de la recherche, il est temps de présenter les résultats obtenus lors de la passation du pré-post test et leurs interprétations.

$\mathrm{N} \quad$ :Nombre des étudiants-maitres.

\section{3}




$\begin{array}{ll}\text { Min } & : \text { Minimum } \\ \text { Max } & : \text { Maximum. } \\ \mathrm{M}_{\mathrm{A}} & \text { :Moyenne Arithmétique } \\ \mathrm{E} & : \text { Ecart type } \\ \mathrm{TN} & : \text { Total des Notes } \\ \mathrm{DL} & : \text { Degrés de liberté } \\ \mathrm{T} & : \text { Valeur de « T } » \text { test } \\ \mathrm{S} & : \text { Significative }\end{array}$

Chaque tableau indique le minimum et le maximum des notes obtenues par les étudiants-maitres dans le pré post test: " le totale et les deux parties du test», la moyenne arithmétique des notes, l'écart type, les degrés de liberté et la valeur de « T » au niveau de 0.001 .

Tableau $n$ : 1 le totale du pré-post test : (50 points)

\begin{tabular}{|l|l|l|l|l|l|l|l|l|}
\hline $\begin{array}{l}\text { Le totale des } \\
\text { notes du test }\end{array}$ & $\mathrm{N}$ & Min & Max & $\mathrm{M}_{\mathrm{A}}$ & $\mathrm{E}$ & $\mathrm{DL}$ & $\mathrm{T}$ & $\mathrm{S}$ \\
\hline Pré-test & 19 & 1 & 5 & 2.57 & 0.96 & 17 & 11 & $\begin{array}{l}\text { Au } \\
\text { niveau de } \\
95 \%\end{array}$ \\
\hline Post-test & 19 & 21 & 50 & 41.1 & 6.0 & 18 & 29 \\
\hline
\end{tabular}

Conclusion : Si on observe les notes des étudiants-maitres du groupe expérimental au pré-post test on constate que les notes du pré-test sont reparties entre $\mathbf{0}$ et $\mathbf{5}$ tandis que celles du post test sont reparties entre $\mathbf{2 1}$ et 48. Ce qui met en relief le développement des étudiants-maitres à l'égard du model proposé, de même, la valeur de « $\mathbf{T}$ » est significative au niveau de $\mathbf{0 . 0 0 1}$ ce qui met en valeur la progression dû à l'application du model dans sa note totale sur le niveau des étudiants-maitres, ainsi l'hypothèse de la recherche est réalisée.

\section{Tableau $n$ :2 la partie théorique du pré-post test : ( 25 points)}

\begin{tabular}{|c|c|c|c|c|c|c|c|c|}
\hline $\begin{array}{l}\text { Les notes de la } \\
\text { partie théorique }\end{array}$ & $\mathbf{N}$ & Min & Max & $\mathbf{M}_{\mathbf{A}}$ & $\mathbf{E}$ & DL & $\mathbf{T}$ & $\mathbf{S}$ \\
\hline Pré-test & 19 & 1 & 5 & 2.57 & 0.96 & 18 & 11.6 & \multirow{2}{*}{$\begin{array}{l}\text { Au niveau de } \\
95 \%\end{array}$} \\
\hline Post-test & 19 & 19 & 25 & 21,73 & 1.66 & 18 & 57 & \\
\hline
\end{tabular}

Conclusion : $\mathrm{Si}$ on observe les notes des étudiants maitres du groupe expérimental au pré-post test on constate que les notes de la partie theorique du pré-test sont reparties entre $\mathbf{0}$ et $\mathbf{5}$ tandis que celles du post test sont reparties entre $\mathbf{1 9}$ et $\mathbf{2 5}$. Ce qui met en relief le développement des étudiants maitres à l'égard de la partie théorique du model proposé, de même, la valeur de « $\mathbf{T}$ » est significative au niveau de $\mathbf{0 . 0 0 1}$ ce qui met en valeur la progression dû à l'application du model en ce qui concerne la partie théorique concernant l'enseignement du $\mathrm{CO}$ en se basant sur la théorie de la DP.

\section{4}




\section{Tableau $n$ :3 la partie pratique du pré-post test : ( 25 points)}

\begin{tabular}{|l|l|l|l|l|l|l|l|l|}
\hline $\begin{array}{l}\text { Les notes de la } \\
\text { partie pratique } \\
\text { du test }\end{array}$ & N & Min & Max & $\mathbf{M}_{\text {A }}$ & E & DL & T & S \\
\cline { 1 - 7 } Pré-test & 19 & 0 & 0 & 0.00 & 0.00 & 0 & 0 & \multirow{2}{*}{$95 \%$} \\
\cline { 1 - 7 } Post-test & 19 & 0 & 25 & 25 & 5.7 & 18 & 18 & \\
\hline
\end{tabular}

Conclusion : $\mathrm{Si}$ on observe les notes des étudiants maitres du groupe expérimental au pré-post test on constate que les notes du pré-test de toutes les étudiantes sont $\mathbf{0}$ tandis que celles du post test sont reparties entre $\mathbf{0}$ et 20. Ce qui met en relief le développement des étudiants maitres à l'égard de la préparation du modèle , de même, la valeur de « $\mathbf{T}$ » est significative au niveau de $\mathbf{0 . 0 0 1}$ ce qui met en valeur la progression dû à l'application du modèle en ce qui concerne la partie pratique.

\section{Suggestions et recommandations concernant l'oral :}

1. Il faut accorder une place beaucoup plus importante à l'enseignement de l'oral à nos facultés de pédagogie par le genre de l'exposé oral formel,

2. Il faut intégrer des pratiques de lectures pour améliorer et perfectionner l'enseignement de la langue.

3. Les étudiantes doivent faire face à un examen final de communication orale par suite se préparer sur l'une ou l'autre des compétences discursives, communicationnelles ou linguistiques

4. Pour enseigner l'oral en classe, d'une manière efficace, il importe de préparer des ateliers d'apprentissage à caractère formatif pour les enseignants de la langue française première ou deuxième langue étrangère.

5. Entrainer les étudiants des écoles gouvernementales et expérimentales dans des ateliers de lectures orales et écrites ce qui mènent à développer chez eux des habiletés et des compétences durables et transférables.

\section{Bibliographie :}

1. A. De PERETTI L. DREVILLON. (1980), Pratiques pédagogiques et développement de la pensée opératoire, PUF.

2. BRAILLON Anne (2008-2009), La différenciation pédagogique. Pour l'implication des étudiants Institut Supérieur de Formation Pédagogique. 
3. Conseil de l'Europe. (2000), Un Cadre Europeen Commun de Reference pour les Langues. Strasbourg: Didier.

4. Cornaire, C. et Germain, C. (1998), La compréhension orale. Paris CLE International.

5. Cuq, J.-P. et Gruca, I. (2002). Cours de didactique du français langue étrangère et seconde.Grenoble, PUG.

6. Davy EITO, (1999 - 2000). Un essai de pédagogie différenciée: la pédagogie de contrat» Mémoire professionnel réalisé à l'IUFM de la Réunion.

7. DE VECCHI, Gérard, (1992), Aider les élèves à apprendre, Hachette Livre.

8. DUCROT- Jean Michèle (2005) : L'enseignement de la compréhension orale, Didactique de l'oral, du discours en FLE.

9. Eduscol.education.fr : (2006) L'évaluation des compétences orales en langues vivantes - Réflexions Actes du séminaire national Paris.

10. Eveline, (2008), Pour ne pas confondre un "modèle pédagogique" avec une "méthode d'enseignement". Education, Ecole et Pédagogie

11. Gosselin, P. (1990). Vers un modèle de modélisation. Dans L'association internationale de pédagogue expérimentale de langue française section canadienne (Dir.), Les modèles en éducation. Montréal : Edition Noir et Blanc.

12. HUBERT, J.-C. (s.d.). (2011), DE LA PEDAGOGIE DIFFERENCIEE.

13. Khaled Muhammad El Hegazy (2004): Efficacité d'un modèle d'enseignement proposée en vue de développer quelques compétences de la pensée critique chez les étudiants de section française à la faculté de Pédagogie. Université de Hélouân.

14. Lafontaine, L. (2004). L"enseignement -apprentissage des genres oraux à l'école secondaire québécoise. AIRDF, (p. \&é). Québec.

15. Louis LEGRAND, (1984), La différenciation pédagogique, Scarabée, CEMEA, Paris)

16. Mc COMBS, Barbara L. et POPE James E., (2000), Motiver ses élèves.

17. Michèle Verdelhan-Bourgade, (2002), Apprendre à comprendre l'oral en situation de français de scolarisation, IUFM de Montpellier, Didaxis-Dipralang, université Montpellier III Didactiques de l'oral.

18. Molette V. (2010) La différenciation pédagogique en maternelle article support de l'animation pédagogique.

\section{6}


19. Nunan, D. (2002). Listening in Language Learning. In J. C. Richards \& W. A. Renandya (Eds.), Methodology in language teaching. An anthology of current practice, Cambridge: Cambridge University Press.

20. Osada, N. (2004). Listening comprehension research: a brief review of the past thirty years. Dialogue Vol. 3.

21. Perrenoud: $\mathrm{Ph}$ (2005) Différencier : un aide-mémoire en quinze points » In Vivre le primaire (Québec), Presses de l'Université du Québec. N 2.

22. PRZESMYCKI, Halina: La Pédagogie différenciée. Hachette Education. Paris 2011.

23. Rapport de l'Inspection Générale, (1999) La place de l'oral dans les enseignements : de l'école primaire au lycée.

24. Rapport de l'IGEN n 99-023, L'ORAL (1999) : langage, situations La place de l'oral dans les enseignements : de l'école primaire au lycée.

25. Raymond Le Blanc, (2001), in « éducation et francophonie » vol. xxv, no 2. Pour le volet I du programme de sensibilisation aux nouvelles approches pédagogiques de la commission scolaire de la rivière du Nord. France.

26. Rey Alanin et autres (1986), Micro Robert. Dictionnaire de français. Montréal. Canada.

27. Silvia LOPEZ DEL HIERRO, (2010) Relations entre la méthodologie de l'enseignement de la compréhension orale et les représentations didactiques des professeurs de français langue étrangère au Mexique Thèse du doctorat Université de Nancy 2 Paris 3

28. Vandergrift, L. (2007). Recent developments in second and foreign language listening comprehension research. Language Teaching.

29. Wirthner, 1991 Wirthner, M. (1991). L’oral comme expérience de soi et rapport à l'autre. In M. Wirthner, D. Martin et P. Perrenoud.Paris Delachaux et Niestlé.

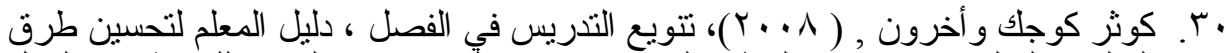

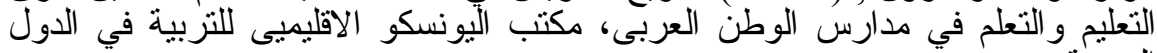
العربية ، بيروت

Site :

31. http://www.differenciationpedagogique.com/questceque/questceque

32. http://WWW.Spirale-edurevue.fr/.../HUBERT_JeanClaude_Dela_ pedagogie

33. http://www.charmeux.fr/blog/index.php

34. http://www.edufle.net/L-Enseignement-de-la-comprehension.html

35. http://www.ecoledeslangues.com/?p=423 


\section{نهوذج هقترح قائم على هدخل "تنويع التدريس" لتنهية ههارات تدريس الفهم

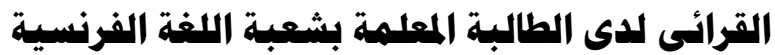

د الهرين صلاح عبد الغني

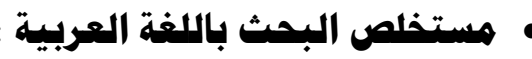

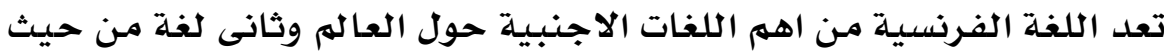

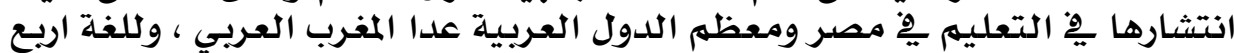

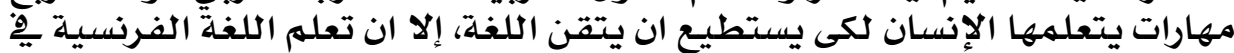

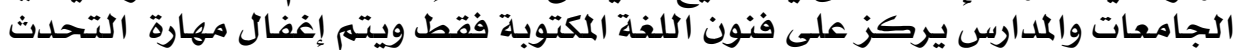

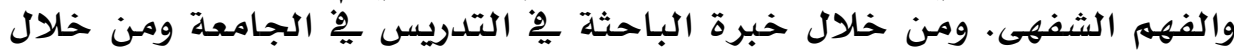

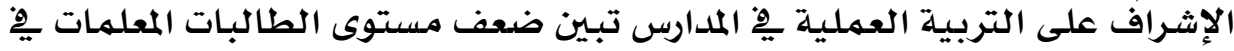

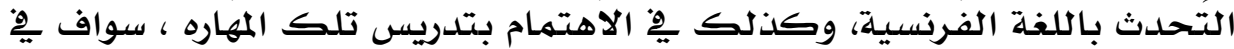

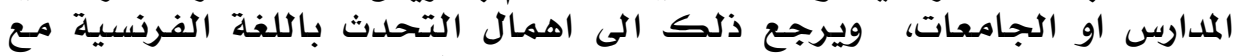

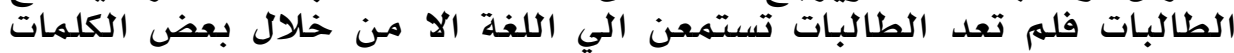

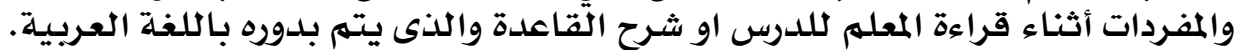

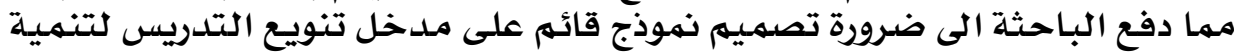

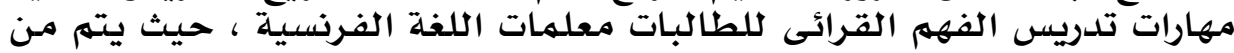

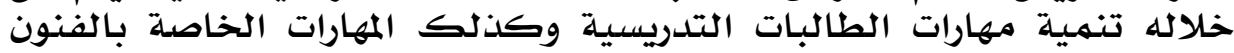

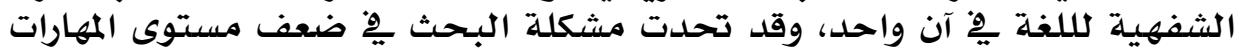

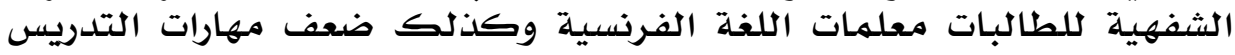

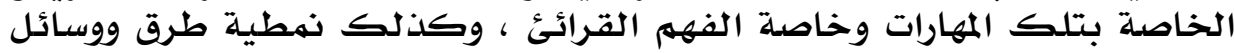

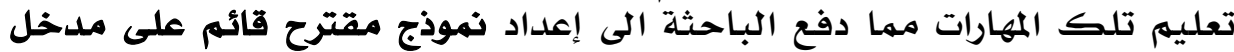

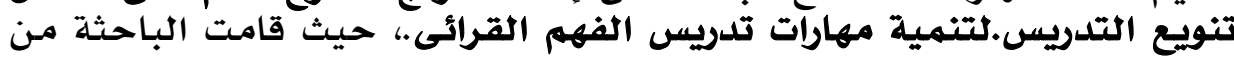

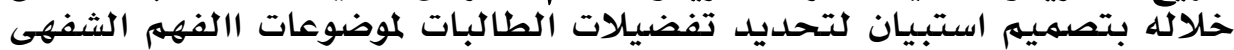

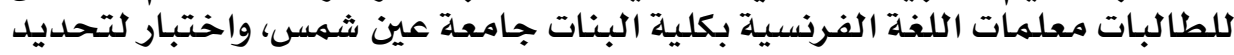

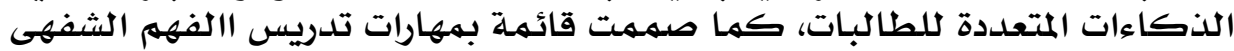

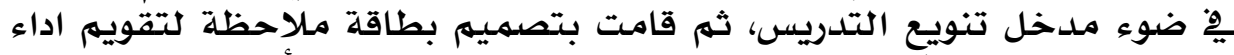

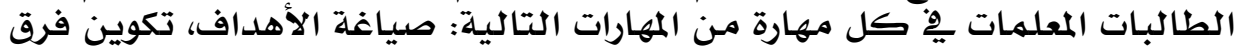

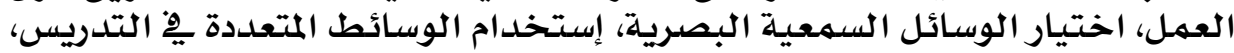

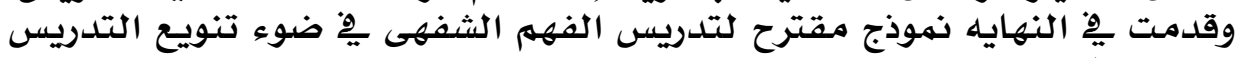

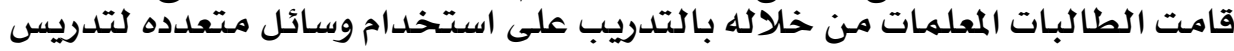

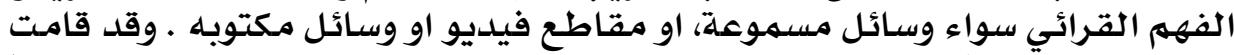

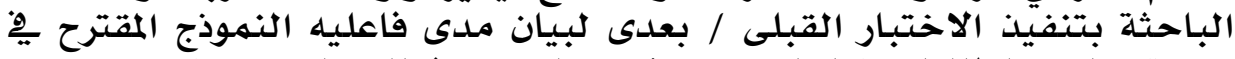

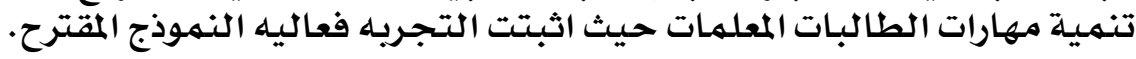

\section{潾潾潾潾}

\section{8}

